\title{
Topology of Random 2-Complexes
}

\author{
D. Cohen - A. Costa $\cdot$ M. Farber $\cdot$ T. Kappeler
}

Received: 22 June 2010 / Revised: 15 April 2011 / Accepted: 23 July 2011 /

Published online: 21 October 2011

(C) Springer Science+Business Media, LLC 2011

\begin{abstract}
We study the Linial-Meshulam model of random two-dimensional simplicial complexes. One of our main results states that for $p \ll n^{-1}$ a random 2-complex $Y$ collapses simplicially to a graph and, in particular, the fundamental group $\pi_{1}(Y)$ is free and $H_{2}(Y)=0$, asymptotically almost surely. Our other main result gives a precise threshold for collapsibility of a random 2-complex to a graph in a prescribed number of steps. We also prove that, if the probability parameter $p$ satisfies $p \gg n^{-1 / 2+\epsilon}$, where $\epsilon>0$, then an arbitrary finite two-dimensional simplicial complex admits a topological embedding into a random 2-complex, with probability tending to one as $n \rightarrow \infty$. We also establish several related results; for example, we show that for $p<c / n$ with $c<3$ the fundamental group of a random 2-complex contains a non-abelian free subgroup. Our method is based on exploiting explicit thresholds (established in the paper) for the existence of simplicial embeddings and immersions of 2-complexes into a random 2-complex.
\end{abstract}

D. Cohen

Department of Mathematics, Louisiana State University, Baton Rouge, LA 70803, USA

e-mail: cohen@math.1su.edu

url: www.math.lsu.edu/ cohen

A. Costa $\cdot$ M. Farber $(\bowtie)$

Mathematics Institute, Zeeman Building, University of Warwick, Coventry CV4 7AL, UK

e-mail: MichaelSFarber@googlemail.com

A. Costa

e-mail: armindocosta@gmail.com

T. Kappeler

Mathematical Insitutte, University of Zurich, Winterthurerstrasse 190, 8057 Zurich, Switzerland e-mail: thomas.kappeler@math.uzh.ch 


\section{Introduction}

The modeling of large systems in applications motivates the development of unconventional geometric and topological notions. Among them are mixed probabilistictopological concepts, such as the Erdős and Rényi random graphs of [6], which are currently used in many applications in engineering and computer science.

More recently, higher dimensional analogs of the Erdős-Rényi model were suggested and studied by Linial-Meshulam in [16] and Meshulam-Wallach in [17]. In these models one generates a random $d$-dimensional complex $Y$ by considering the full $d$-dimensional skeleton of the simplex $\Delta_{n}$ on vertices $\{1, \ldots, n\}$ and retaining $d$-dimensional faces independently with probability $p$. The work of LinialMeshulam and Meshulam-Wallach provides threshold functions for the vanishing of the $(d-1)$-st homology groups of random complexes with coefficients in a finite abelian group. Threshold functions for the vanishing of the $d$-th homology groups were subsequently studied by Kozlov [15].

An interesting class of closed smooth manifolds depending on a large number of random parameters arises as configuration spaces of mechanical linkages with bars of random lengths; see [7,8]. Although the number of homeomorphism types of these manifolds grows extremely fast, their topological characteristics can be predicted with high probability when the number of links tends to infinity.

In this paper, we study the topology of random two-dimensional complexes. The probability space $G\left(\Delta_{n}^{(2)}, p\right)$ of the Linial-Meshulam model of random 2-complexes is defined as follows. Let $\Delta_{n}$ denote the $(n-1)$-dimensional simplex with vertices $\{1,2, \ldots, n\}$. Then $G\left(\Delta_{n}^{(2)}, p\right)$ denotes the set of all two-dimensional subcomplexes

$$
\Delta_{n}^{(1)} \subset Y \subset \Delta_{n}^{(2)},
$$

containing the one-dimensional skeleton $\Delta_{n}^{(1)}$. The probability function $\mathbb{P}$ : $G\left(\Delta_{n}^{(2)}, p\right) \rightarrow \mathbf{R}$ is given by the formula

$$
\mathbb{P}(Y)=p^{f(Y)}(1-p)^{\left(\begin{array}{c}
n \\
3
\end{array}\right)-f(Y)}, \quad Y \in G\left(\Delta_{n}^{(2)}, p\right),
$$

where $f(Y)$ denotes the number of faces in $Y$. In other words, each of the twodimensional simplexes of $\Delta_{n}^{(2)}$ is included in a random 2-complex $Y$ with probability $p$, independently of the other 2-simplexes. As in the case of random graphs, $0<p<1$ is a probability parameter which may depend on $n$. The model $G\left(\Delta_{n}^{(2)}, p\right)$ includes all finite two-dimensional simplicial complexes containing the full 1-skeleton $\Delta_{n}^{(1)}$; however, the likelihood of various topological phenomena is dependent on the value of $p$. The theory of deterministic 2-complexes itself is a rich and active field of current research with many challenging open questions; see [12].

The fundamental group of a random 2-complex $Y \in G\left(\Delta_{n}^{(2)}, p\right)$ was investigated by Babson, Hoffman, and Kahle [2]. They showed that for ${ }^{1}$

$$
p \gg n^{-1 / 2} \cdot(3 \log n)^{1 / 2}
$$

\footnotetext{
${ }^{1}$ Recall that the symbol $a_{n} \ll b_{n}$ means that $a_{n}>0$ and $a_{n} / b_{n} \rightarrow 0$ as $n \rightarrow \infty$.
} 
the group $\pi_{1}(Y)$ vanishes asymptotically almost surely (a.a.s). ${ }^{2}$ These authors use notions of negative curvature due to Gromov to study the nontriviality and hyperbolicity of $\pi_{1}(Y)$ for

$$
p \ll n^{-1 / 2-\epsilon} .
$$

In this paper we prove the following theorem.

Theorem 1 If the probability parameter $p$ satisfies

$$
p \ll n^{-1}
$$

then a random 2-complex $Y \in G\left(\Delta_{n}^{(2)}, p\right)$ collapses simplicially to a graph, a.a.s. In particular, the fundamental group $\pi_{1}(Y)$ is free, and for any coefficient group $G$ one has $\mathrm{H}_{2}(Y ; G)=0$, a.a.s.

Loosely speaking, Theorem 1 combines with previously known results to suggest that a random 2-complex with vanishing two-dimensional homology is homotopically one dimensional.

We also prove a modification of the above theorem having a slightly stronger conclusion under more restrictive assumptions.

Theorem 2 (a) Iffor some $k \geq 1$ the probability parameter $p$ satisfies

$$
p \ll n^{-1-\frac{2}{k+1}},
$$

then a random 2-complex $Y \in G\left(\Delta_{n}^{(2)}, p\right)$ is collapsible to a graph in at most $k$ steps, asymptotically almost surely (a.a.s). (b) If for some $k \geq 1$ the probability parameter p satisfies

$$
p \gg n^{-1-\frac{1}{3 \cdot 2^{k-1}-1}},
$$

then $Y$ is not collapsible to a graph in $k$ or fewer steps, a.a.s.

We conjecture that similar results hold for $d$-dimensional random complexes in the Meshulam-Wallach model [17]; i.e., for $p \ll n^{-1}$ a random $d$-dimensional complex collapses simplicially to a $(d-1)$-dimensional subcomplex. This would strengthen a theorem of D. Kozlov [15].

Another major result of this paper that is proven in Sect. 8 states the following.

Theorem 3 Assume that for some $\epsilon>0$ the probability parameter $p$ satisfies $p \gg n^{-1 / 2+\epsilon}$. Let $S$ be an arbitrary simplicial finite 2-complex. Then $S$ admits a topological embedding into a random 2-complex $Y \in G\left(\Delta_{n}^{(2)}\right.$, p), a.a.s.

By a topological embedding $S \rightarrow Y$ we mean a simplicial embedding of a subdivision of $S$ into $Y$.

\footnotetext{
${ }^{2}$ We use the abbreviation a.a.s. for the phrase "asymptotically almost surely."
} 
The method of this paper is based on studying simplicial embeddings and immersions of polyhedra into random 2-complexes. We analyze in detail the numerical invariants $\mu(S)$ and $\tilde{\mu}(S)$, defined in Sect. 3, which play a crucial role in the questions about the existence of embeddings and immersions. We also discuss the notion of balanced triangulations, a generalization of the notion of a balanced graph in the random graph theory. We prove that any triangulation of a closed surface is balanced, although surfaces with boundary (even disks) admit unbalanced triangulations.

Among some other results presented in this paper we may mention the statement that for $p<c / n$, where $c<3$, the fundamental group of a random 2-complex contains a non-abelian free subgroup, a.a.s. We also prove that for $p>c / n$ with $c>3$ the second homology group of a random 2-complex is nontrivial a.a.s; this strengthens a result of D. Kozlov [15].

The proof of Theorem 1 is given in Sect. 5, while Theorem 2 is proved at the very end of the paper. A key role in the proof of the latter is played by Theorem 46, which roughly states that there is a finite list of forbidden 2-complexes so that an arbitrary 2-complex is collapsible to a graph in $k$ steps if and only if it does not contain any of the 2-complexes from this list. This enables us to reduce the collapsibility problem addressed in Theorem 2 to the containment problem for random complexes.

The results presented in this paper were originally announced in two preprints [3] and [4].

After this paper was submitted for publication an interesting preprint of Aronshtam, Linial, Łuczak, and Meshulam [1] appeared. This preprint improves the threshold of Kozlov [15] on the vanishing of the top-dimensional homology of a random complex. The result of [1] gives more information on the constant $c$ which appears in Theorem 6 below.

\section{Preliminaries}

\subsection{Basic Definitions}

For the convenience of the reader we collect in this section the definitions of basic combinatorial notions related to two-dimensional complexes which will be used in this paper.

Let $Y$ be a finite two-dimensional simplicial complex. An edge of $Y$ is called free if it is included in exactly one 2-simplex.

The boundary $\partial Y$ is defined as the union of free edges. We say that a 2-complex $Y$ is closed if $\partial Y=\emptyset$.

A 2-complex $Y$ is called pure if every maximal simplex is two dimensional. By the pure part of a 2-complex we mean the maximal pure subcomplex, i.e., the union of all 2-simplexes.

Let $Y$ be a simplicial 2-complex and let $\sigma$ and $\tau$ be two 2-simplexes of $Y$. We say that $\sigma$ and $\tau$ are adjacent if they intersect in an edge. The distance between $\sigma$ and $\tau, d_{Y}(\sigma, \tau)$, is the minimal integer $k$ such that there exists a sequence of 2-simplexes $\sigma=\sigma_{0}, \sigma_{1}, \ldots, \sigma_{k}=\tau$ with the property that $\sigma_{i}$ is adjacent to $\sigma_{i+1}$ for every $0 \leq i<k$. (If no such sequence exists, then $d_{Y}(\sigma, \tau)=\infty$.) The diameter 
$\operatorname{diam}(Y)$ is defined as the maximal value of $d_{Y}(\sigma, \tau)$ taken over pairs of 2-simplexes of $Y$.

A simplicial 2-complex is strongly connected if it has a finite diameter.

A simplicial 2-complex has degree $\leq r$ if every edge is incident to at most $r$ 2-simplexes.

A pseudo-surface is a finite, pure, strongly connected two-dimensional simplicial complex of degree at most 2 (i.e., every edge is included in at most two 2-simplexes).

More generally, for an integer $r>0$, an $r$-pseudo-surface is a finite, pure, strongly connected two-dimensional simplicial complex of degree at most $r$.

Simplicial Collapse

Let $Y$ be a 2-complex. A 2-simplex of $Y$ is called free if at least one of its edges is free. Let $\sigma_{1}, \ldots, \sigma_{k}$ be all free 2-simplexes in $Y$, and let $e_{1}, \ldots, e_{k}$ be free edges with $e_{i} \subset \sigma_{i}$. We say that the complex

$$
Y^{\prime}=Y-\bigcup_{i=1}^{k} \operatorname{int}\left(\sigma_{i}\right)-\bigcup_{i=1}^{k} \operatorname{int}\left(e_{i}\right)
$$

is obtained from $Y$ by collapsing all free 2-simplexes. Clearly $Y^{\prime} \subset Y$ is a deformation retract. The operation $Y \searrow Y^{\prime}$ is called a simplicial collapse. Note that $Y^{\prime}$ is not uniquely determined if one of the free simplexes of $Y$ has two free edges; however, the pure part of $Y^{\prime}$ (i.e., the union of 2-simplexes of $Y^{\prime}$ ) is uniquely determined.

This process can be iterated $Y^{\prime} \searrow Y^{\prime \prime}, Y^{\prime \prime} \searrow Y^{\prime \prime \prime}$, etc. We denote $Y=Y^{(0)}$, $Y^{\prime}=Y^{(1)}, Y^{\prime \prime}=Y^{(2)}$ etc. The sequence of subcomplexes $Y^{(0)} \supset Y^{(1)} \supset Y^{(2)} \supset \cdots$ is decreasing, and there are two possibilities: either (a) for some $k$, the complex $Y^{(k)}$ is one dimensional (a graph), or (b) for some $k$, the complex $Y^{(k)}$ is two dimensional and closed, i.e., $\partial Y^{(k)}=\emptyset$.

Definition 4 We say that $Y$ is collapsible to a graph in at most $k$ steps if $Y^{(k)}$ is a graph. We say that $Y$ is collapsible to a graph in $k$ steps if $Y^{(k)}$ is a graph and $\operatorname{dim} Y^{(k-1)}=2$.

Observe that if $Y$ is collapsible to a graph in at most $k$ steps, then any simplicial subcomplex $S \subset Y$ is also collapsible to a graph in at most $k$ steps. At each step one removes the free triangles in $Y^{(i)}$ which belong to $S$.

\section{The Fundamental Group and the Second Betti Number}

In this section we analyze the fundamental group and the second Betti number of a random 2-complex using mainly information provided by the Euler characteristic. The results of this section are specific for two-dimensional random complexes. 
Theorem 5 Suppose that $p<c n^{-1}$, where $c<3$. Then the fundamental group $\pi_{1}(Y)$ of a random 2-complex $Y \in G\left(\Delta_{n}^{(2)}, p\right)$ contains a non-abelian free subgroup with probability at least $1-\lambda^{n^{2}}$, for all large enough $n$, where

$$
\lambda=\exp \left(-\frac{1}{8}\left(1-\frac{c}{3}\right)^{2}\right)
$$

$0<\lambda<1$. In particular, $\pi_{1}(Y)$ contains a free subgroup on two generators, a.a.s.

Proof The Euler characteristic of $Y \in G\left(\Delta_{n}^{(2)}, p\right)$ can be written as

$$
\chi(Y)=n-\left(\begin{array}{l}
n \\
2
\end{array}\right)+f_{2}(Y)=f_{2}(Y)+1-\left(\begin{array}{c}
n-1 \\
2
\end{array}\right)
$$

where $f_{2}(Y)$ denotes the number of 2-simplexes in $Y$. Clearly, the function $f_{2}$ : $G\left(\Delta_{n}^{(2)}, p\right) \rightarrow \mathbf{Z}$ coincides with the sum of random variables

$$
f_{2}=\sum_{\sigma} I_{\sigma}
$$

where $\sigma$ runs over 2-simplexes $\left(i, j, k\right.$ ) (with $1 \leq i<j<k \leq n$ ) and $I_{\sigma}(Y)=1$ iff $\sigma$ is included in $Y$; otherwise $I_{\sigma}(Y)=0$. Each $I_{\sigma}$ is a Bernoulli random variable with parameter $p$ and $f_{2}$ has binomial distribution

$$
\mathbb{P}\left(f_{2}(Y)=k\right)=\left(\begin{array}{c}
\left(\begin{array}{c}
n \\
3
\end{array}\right) \\
k
\end{array}\right) p^{k}(1-p)^{\left(\begin{array}{c}
n \\
3
\end{array}\right)-k},
$$

where $k=0,1,2, \ldots,\left(\begin{array}{l}n \\ 3\end{array}\right)$. The expectation $\mathbb{E}\left(f_{2}\right)$ equals $p\left(\begin{array}{l}n \\ 3\end{array}\right)$. Using inequality (2.5) from [13] we find that for any $t \geq 0$

$$
\mathbb{P}\left(f_{2} \geq p\left(\begin{array}{l}
n \\
3
\end{array}\right)+t\right) \leq \exp \left(-\frac{t^{2}}{2\left(p\left(\begin{array}{l}
n \\
3
\end{array}\right)+t / 3\right)}\right) .
$$

Consider inequality (2) with

$$
t=\left(1-\frac{p n}{3}\right)\left(\begin{array}{c}
n-1 \\
2
\end{array}\right)-1 .
$$

We observe that: (i) the assumption $p n<c<3$ implies that $t>0$ for large $n$, and (ii) the inequality $f_{2}(Y) \geq p\left(\begin{array}{l}n \\ 3\end{array}\right)+t$ is equivalent to the inequality $\chi(Y) \geq 0$. We thus obtain from (2)

$$
\mathbb{P}(\chi(Y) \geq 0) \leq \exp \left(-\frac{t^{2}}{2\left(p\left(\begin{array}{l}
n \\
3
\end{array}\right)+t / 3\right)}\right)
$$

and from (3), for $n \geq 3$,

$$
p\left(\begin{array}{l}
n \\
3
\end{array}\right)+\frac{t}{3} \leq \frac{1}{3}\left(\frac{2 c}{3}+1\right)\left(\begin{array}{c}
n-1 \\
2
\end{array}\right)-\frac{1}{3} \leq\left(\begin{array}{c}
n-1 \\
2
\end{array}\right)
$$


as $c<3$. Thus one gets for $n$ sufficiently large

$$
\begin{aligned}
\frac{t^{2}}{2\left(p\left(\begin{array}{c}
n \\
3
\end{array}\right)+t / 3\right)} & \geq \frac{1}{2} \frac{\left[\left(1-\frac{p n}{3}\right)\left(\begin{array}{c}
n-1 \\
2
\end{array}\right)-1\right]^{2}}{\left(\begin{array}{c}
n-1 \\
2
\end{array}\right)} \\
& \geq \frac{1}{3}\left(1-\frac{c}{3}\right)^{2} \cdot\left(\begin{array}{c}
n-1 \\
2
\end{array}\right) \\
& \geq \frac{1}{8}\left(1-\frac{c}{3}\right)^{2} \cdot n^{2} .
\end{aligned}
$$

Therefore, by the definition of $\lambda$,

$$
\mathbb{P}(\chi(Y) \geq 0) \leq \exp \left(-\frac{1}{8}\left(1-\frac{c}{3}\right)^{2} n^{2}\right)=\lambda^{n^{2}}
$$

and thus

$$
\mathbb{P}(\chi(Y)<0) \geq 1-\lambda^{n^{2}} .
$$

Theorem 5 now follows from a theorem proven in [9] which states: If the Euler characteristic of a finite connected two-dimensional polyhedron $Y$ is negative, $\chi(Y)<0$, then $\pi_{1}(Y)$ contains a non-abelian free subgroup.

This completes the proof.

Theorem 6 Suppose that $p>c n^{-1}$, where now $c>3$. Then for a random twodimensional complex $Y \in G\left(\Delta_{n}^{(2)}, p\right)$ one has $H_{2}(Y ; \mathbf{Z}) \neq 0$ with probability at least $1-\mu^{n^{2}}$, for all large enough $n$, where

$$
\mu=\exp \left(-\frac{1}{8}\left(\frac{c}{3}-1\right)\right)
$$

$0<\mu<1$. In particular, ${ }^{3} H_{2}(Y ; \mathbf{Z}) \neq 0$, a.a.s.

Proof The proof is very similar to the one of Theorem 5 and also uses the Euler characteristic. Clearly, $\chi(Y)=1-b_{1}(Y)+b_{2}(Y)$ (where $b_{i}(Y)$ denotes the $i$-dimensional Betti number, $b_{i}(Y)=\operatorname{rk} H_{i}(Y ; \mathbf{Z})$ ). Thus $\chi(Y)>1$ implies $b_{2}(Y)>0$. We will estimate from above the probability of the complementary event $\chi(Y) \leq 1$.

Using inequality (2.6) from [13] one has for any $t \geq 0$

$$
\mathbb{P}\left(f_{2} \leq p\left(\begin{array}{l}
n \\
3
\end{array}\right)-t\right) \leq \exp \left(-\frac{t^{2}}{2 p\left(\begin{array}{l}
n \\
3
\end{array}\right)}\right) .
$$

Now choose

$$
t=\left(\frac{p n}{3}-1\right) \cdot\left(\begin{array}{c}
n-1 \\
2
\end{array}\right)
$$

\footnotetext{
${ }^{3}$ Note that $H_{2}(Y ; \mathbf{Z}) \neq 0$ implies that $H_{2}(Y ; G) \neq 0$ for any coefficient group $G \neq 0$.
} 
Since $p n>c>3$ we have

$$
t>\left(\frac{c}{3}-1\right) \cdot\left(\begin{array}{c}
n-1 \\
2
\end{array}\right)>0 .
$$

The inequality $f_{2}(Y) \leq p\left(\begin{array}{l}n \\ 3\end{array}\right)-t$ is equivalent to $\chi(Y) \leq 1$. Thus we obtain

$$
\mathbb{P}(\chi(Y) \leq 1) \leq \exp \left(-\frac{t^{2}}{2 p\left(\begin{array}{l}
n \\
3
\end{array}\right)}\right)
$$

and, for $n$ sufficiently large,

$$
\begin{aligned}
\frac{t^{2}}{2 p\left(\begin{array}{c}
n \\
3
\end{array}\right)} & \geq \frac{\left(\frac{p n}{3}-1\right)^{2} \cdot\left(\begin{array}{c}
n-1 \\
2
\end{array}\right)}{2 \frac{p n}{3}} \\
& \geq \frac{1}{2}\left(\frac{p n}{3}-1\right) \cdot\left(\begin{array}{c}
n-1 \\
2
\end{array}\right) \\
& \geq \frac{1}{8}\left(\frac{c}{3}-1\right) \cdot n^{2} .
\end{aligned}
$$

Finally, by the definition of $\mu$,

$$
\mathbb{P}\left(b_{2}(Y)=0\right) \leq \mathbb{P}(\chi(Y) \leq 1) \leq \mu^{n^{2}} .
$$

This completes the proof.

Next we consider the critical case $p=3 / n$.

Theorem 7 Assume that $p=\frac{3}{n}$. Then for any $\epsilon>0$ there exists $N$ such that for all $n>N$ the probability of each of the following statements (a) and (b) concerning a random 2-complex $Y \in G\left(\Delta_{n}^{(2)}, p\right)$ is greater than $\frac{1}{2}-\epsilon$ :

(a) the fundamental group $\pi_{1}(Y)$ contains a noncommutative free subgroup;

(b) $\mathrm{H}_{2}(Y ; \mathbf{Z}) \neq 0$.

It is not known if (a) and (b) exclude each other; one may ask about the probability that, asymptotically, (a) and (b) hold simultaneously.

Proof In the case when $p=3 / n$ one has $\mathbb{E}\left(f_{2}\right)=\left(\begin{array}{c}n-1 \\ 2\end{array}\right)$ and $\mathbb{E}(\chi)=1$ where $f_{2}, \chi$ : $G\left(\Delta_{n}^{(2)}, p\right) \rightarrow \mathbf{Z}$ are as above. From the de Moivre-Laplace integral theorem [20], p. 62 , it follows that

$$
\mathbb{P}\left(f_{2}>\left(\begin{array}{c}
n-1 \\
2
\end{array}\right)\right) \sim \frac{1}{\sqrt{2 \pi}} \int_{0}^{\infty} e^{-x^{2} / 2} d x=\frac{1}{2}
$$

and

$$
\mathbb{P}\left(f_{2} \leq\left(\begin{array}{c}
n-1 \\
2
\end{array}\right)-2\right) \sim \frac{1}{\sqrt{2 \pi}} \int_{-\infty}^{b} e^{-x^{2} / 2} d x \sim \frac{1}{2},
$$


where $b$ can be found from the equation $b \sqrt{\left(\begin{array}{c}n \\ 3\end{array}\right) p(1-p)}=-2$, i.e.,

$$
b=-\frac{2}{\sqrt{\left(\begin{array}{c}
n-1 \\
2
\end{array}\right) \cdot\left(1-\frac{3}{n}\right)}} \sim 0 .
$$

By (1), the inequality $f_{2}(Y)>\left(\begin{array}{c}n-1 \\ 2\end{array}\right)$ is equivalent to $\chi(Y)>1$ and the inequality $f_{2}(Y)<\left(\begin{array}{c}n-1 \\ 2\end{array}\right)-1$ is equivalent to $\chi(Y)<0$. Thus we see that

$$
\mathbb{P}(\chi(Y)>1) \sim \frac{1}{2}, \quad \text { and } \quad \mathbb{P}(\chi(Y)<0) \sim \frac{1}{2}
$$

and thus, for any given $\epsilon>0$,

$$
\begin{gathered}
\mathbb{P}\left(\pi_{1}(Y) \supset F_{2}\right) \geq \mathbb{P}(\chi(Y)<0) \geq \frac{1}{2}-\epsilon, \\
\mathbb{P}\left(b_{2}(Y)>0\right) \geq \mathbb{P}(\chi(Y)>1) \geq \frac{1}{2}-\epsilon
\end{gathered}
$$

for sufficiently large $n$. Here $F_{2}$ denotes the free group with two generators.

\section{Simplicial Embeddings and Immersions}

In this section we consider the containment problem for subcomplexes of random two-dimensional complexes, which is similar to the containment problem for random graphs; see [13], Chapt. 3. We also study simplicial immersions, which are more general than simplicial embeddings.

Let $S$ be a two-dimensional finite simplicial complex. We denote by $v=v_{S}$ and $f=f_{S}$ the numbers of vertices and faces of $S$ respectively. The set of vertices of $S$ is denoted by $V(S)$. We assume that $S$ is fixed, i.e., independent of $n$.

Definition 8 A simplicial embedding $g: S \hookrightarrow Y$, where $Y \in G\left(\Delta_{n}^{(2)}, p\right)$ is a random 2-complex, is defined as an injective map of the set of vertices $V(S)$ of $S$ into the set of vertices $\{1, \ldots, n\}$ of $Y$ satisfying the following condition: for any triple of distinct vertices $u_{1}, u_{2}, u_{3} \in V(S)$ which span a simplex in $S$, the corresponding points $g\left(u_{1}\right), g\left(u_{2}\right), g\left(u_{3}\right) \in\{1, \ldots, n\}$ span a face of $Y$.

Next we define the following slightly more general notion.

Definition 9 A simplicial immersion $g: S \leftrightarrow Y$ into a random 2-complex $Y \in$ $G\left(\Delta_{n}^{(2)}, p\right)$ is defined as a map of the set of vertices $V(S)$ of $S$ into the set of vertices $\{1, \ldots, n\}$ of $Y$ satisfying the following two conditions:

(a) for any triple of distinct vertices $u_{1}, u_{2}, u_{3} \in V(S)$ which span a 2-simplex in $S$, the corresponding points $g\left(u_{1}\right), g\left(u_{2}\right), g\left(u_{3}\right) \in\{1, \ldots, n\}$ are pairwise distinct and span a face of $Y$;

(b) for any pair of distinct 2-simplexes $\sigma$ and $\sigma^{\prime}$ of $S$, the corresponding 2-simplexes $g(\sigma)$ and $g\left(\sigma^{\prime}\right)$ of $Y$ are distinct. 
Note that a simplicial immersion $g: S \leftrightarrow Y$ is not necessarily injective on the set of vertices $V(S)$, but any pair of vertices $u_{1}, u_{2} \in V(S)$ with $g\left(u_{1}\right)=g\left(u_{2}\right)$ cannot lie in a 2-simplex of $S$. We also require that distinct 2-simplexes of $S$ are mapped to distinct 2-simplexes of $Y$.

If $g: S \leftrightarrow Y$ is a simplicial immersion, then for any subcomplex $S^{\prime} \subset S$ the restriction $g \mid S^{\prime}$ is also a simplicial immersion $S^{\prime} \uparrow \rightarrow Y$.

Lemma 10 The probability that a two-dimensional simplicial complex $S$ with $v$ vertices and $f$ faces admits a simplicial immersion into a random 2-complex $Y \in$ $G\left(\Delta_{n}^{(2)}, p\right)$ is less than or equal to $n^{v} p^{f}$, i.e.,

$$
\mathbb{P}(S \leftrightarrow Y) \leq n^{v} p^{f} .
$$

Proof For a map $g: V(S) \rightarrow\{1, \ldots, n\}$ denote by $J_{g}: G\left(\Delta_{n}^{(2)}, p\right) \rightarrow\{0,1\}$ the random variable such that $J_{g}(Y)=1$ if and only if $g$ determines a simplicial immersion $S \leftrightarrow Y$, i.e., if the conditions of Definition 9 are satisfied. Clearly, the expectation $\mathbb{E}\left(J_{g}\right)$ equals $p^{f}$. The random variable $X_{S}=\sum_{g} J_{g}$ counts the number of simplicial immersions $S \leftrightarrow Y$, where $g$ runs over all maps $V(S) \rightarrow\{1, \ldots, n\}$. Thus

$$
\mathbb{E}\left(X_{S}\right)=\sum_{g} \mathbb{E}\left(J_{g}\right) \leq n^{v} \cdot p^{f}
$$

and

$$
\mathbb{P}(S \uparrow Y)=\mathbb{P}\left(X_{S}>0\right) \leq \mathbb{E}\left(X_{S}\right) \leq n^{v} p^{f},
$$

by the first moment method.

Next we define a useful numerical invariant which was also mentioned in [2].

Definition 11 For a simplicial 2-complex $S$ let $\mu(S)$ denote

$$
\mu(S)=\frac{v}{f} \in \mathbf{Q},
$$

where $v=v_{S}$ and $f=f_{S}$ are the numbers of vertices and faces in $S$.

Corollary 12 If the probability parameter p satisfies

$$
p \ll n^{-\mu(S)}
$$

then the 2-complex $S$ admits no simplicial immersions into a random 2-complex $Y \in$ $G\left(\Delta_{n}^{(2)}, p\right)$, a.a.s.

Proof The assumption $p \ll n^{-\mu(S)}$ means that $p n^{\mu(S)} \rightarrow 0$ as $n \rightarrow \infty$. Then $n^{v} p^{f} \rightarrow 0$ and the result now follows from Lemma 10 .

As an example consider a simplicial graph $\Gamma$ and the cone over it $S=C(\Gamma)$. One has $v_{S}=v_{\Gamma}+1$ and $f_{S}=e_{\Gamma}$. Therefore

$$
\mu(S)=\frac{v_{\Gamma}+1}{e_{\Gamma}} .
$$

Using Corollary 12 we obtain the following. 
Corollary 13 If a graph $\Gamma$ satisfies $\chi(\Gamma)<0$ then $\mu(S) \leq 1$ where $S=C(\Gamma)$ is the cone over $\Gamma$. Therefore, if, $p \ll n^{-1}$, then the cone $S=C(\Gamma)$ with $\chi(\Gamma)<0$ admits no simplicial immersions into a random 2-complex $Y \in G\left(\Delta_{n}^{(2)}, p\right)$, a.a.s.

This result will be used later in this paper.

Definition 14 Let $S$ be a finite two-dimensional simplicial complex. Define

$$
\tilde{\mu}(S)=\min _{S^{\prime} \subset S} \mu\left(S^{\prime}\right),
$$

where the minimum is formed over all subcomplexes $S^{\prime} \subset S$ or, equivalently, over all pure subcomplexes $S^{\prime} \subset S$.

Note that the invariant $\tilde{\mu}$ is monotone decreasing: if $S$ is a subcomplex of $T$ then

$$
\tilde{\mu}(S) \geq \tilde{\mu}(T) .
$$

The following result complements Corollary 12 .

Theorem 15 Let $S$ be a finite simplicial complex.

(A) If $p \ll n^{-\tilde{\mu}(S)}$ then the probability that $S$ admits a simplicial immersion into a random 2-complex $Y \subset G(n, p)$ tends to zero as $n \rightarrow \infty$.

(B) If $p \gg n^{-\tilde{\mu}(S)}$ then the probability that $S$ admits a simplicial embedding into a random 2-complex $Y \subset G(n, p)$ tends to one as $n \rightarrow \infty$.

Proof Let $S^{\prime} \subset S$ be a subcomplex such that $\mu\left(S^{\prime}\right)=\tilde{\mu}(S) \leq \mu(S)$. Then

$$
\mathbb{P}(S \leftrightarrow Y) \leq \mathbb{P}\left(S^{\prime} \leftrightarrow Y\right)
$$

and $\mathbb{P}\left(S^{\prime} \leftrightarrow Y\right)$ tends to zero assuming that $p \ll n^{-\mu\left(S^{\prime}\right)}=n^{-\tilde{\mu}(S)}$ by Corollary 12 . This proves the statement (A).

The following arguments prove the statement (B). Let $v$ denote the number of vertices of $S$. A simplicial embedding of $S$ into $Y$ is defined by an injective map $g: V(S) \rightarrow\{1, \ldots, n\}$ where $V(S)$ is the set of vertices of $S$. The function $X_{S}=\sum_{g} J_{g}: G\left(\Delta_{n}^{(2)}, p\right) \rightarrow \mathbf{Z}$ counts the number of simplicial embeddings; here $g: V(S) \rightarrow\{1, \ldots, n\}$ runs over all injective maps and $J_{g}$ denotes the random variable defined as in the proof of Lemma 10.

For a pair of injective maps $g, g^{\prime}: V(S) \rightarrow\{1, \ldots, n\}$ consider the pure subcomplex $H=H\left(g, g^{\prime}\right) \subset S$ which is defined as the union of all 2-simplexes $\sigma \subset S$ with the property $g(\sigma) \subset g^{\prime}(S)$. Note that the product random variable $J_{g} J_{g^{\prime}}$ has the expectation

$$
\mathbb{E}\left(J_{g} J_{g^{\prime}}\right)=p^{2 f-f_{H}},
$$

where $f=f_{S}$ is the number of faces of $S$ and $f_{H}$ is the number of 2-simplexes in $H$.

Now we fix a pure subcomplex $H \subset S$ and consider all ordered pairs of injective maps $g, g^{\prime}: V(S) \rightarrow\{1, \ldots, n\}$ with $H\left(g, g^{\prime}\right)=H$. The number $N$ of such pairs $g, g^{\prime}$ satisfies

$$
N \leq C_{H} n^{2 v-v_{H}}
$$


for some constant $C_{H}>0$ depending on $H$.

The variance of $X_{S}$ can be estimated as follows:

$$
\begin{aligned}
\operatorname{Var}\left(X_{S}\right) & =\mathbb{E}\left(X_{S}^{2}\right)-\mathbb{E}\left(X_{S}\right)^{2} \\
& =\sum_{g, g^{\prime}}\left[\mathbb{E}\left(J_{g} J_{g^{\prime}}\right)-\mathbb{E}\left(J_{g}\right) \mathbb{E}\left(J_{g^{\prime}}\right)\right] \\
& \leq \sum_{H \subset S} C_{H} n^{2 v-v_{H}}\left[p^{2 f-f_{H}}-p^{2 f}\right] \\
& =\sum_{H \subset S} C_{H} n^{2 v-v_{H}} p^{2 f-f_{H}}\left[1-p^{f_{H}}\right] .
\end{aligned}
$$

Since for $n$ sufficiently large,

$$
\mathbb{E}\left(X_{S}\right)=\left(\begin{array}{l}
n \\
v
\end{array}\right) v ! \cdot p^{f} \geq \frac{1}{2} \cdot n^{v} p^{f},
$$

it follows that

$$
\frac{\operatorname{Var}\left(X_{S}\right)}{\mathbb{E}\left(X_{S}\right)^{2}} \leq 4 \cdot(1-p) \cdot \sum_{H \subset S}\left(f_{H} C_{H}\right) \cdot\left(n^{v_{H}} p^{f_{H}}\right)^{-1} .
$$

Now, if $p \gg n^{-\tilde{\mu}(S)}$ then $n^{v_{H}} p^{f_{H}} \rightarrow \infty$ for any pure subcomplex $H \subset S$ and therefore each term in the sum above tends to zero. Thus, using the Chebyshev inequality

$$
\mathbb{P}\left(X_{S}=0\right) \leq \frac{\operatorname{Var}\left(X_{S}\right)}{\mathbb{E}\left(X_{S}\right)^{2}},
$$

we see that $\mathbb{P}\left(X_{S}=0\right) \rightarrow 0$ as $n \rightarrow \infty$. This implies statement (B).

The above proof also gives the following quantitative statement.

Corollary 16 Let $S$ be a fixed 2-complex. Then the probability $\mathbb{P}(S \not \subset Y)$ that $S$ is not embeddable into a random 2-complex $Y$ can be estimated by

$$
\mathbb{P}(S \not \subset Y) \leq C \cdot(1-p) \cdot \sum_{H \subset S, f_{H}>0}\left(n^{v_{H}} p^{f_{H}}\right)^{-1},
$$

where $C$ is a constant depending on $S$ and $H$ runs through all pure subcomplexes of $S$.

\section{Proof of Theorem 1}

In this section we prove Theorem 1 stated in the Introduction.

Note that the assumptions and conclusions of Theorem 1 are stronger than those of Theorem 5. One may also compare Theorem 1 with Theorem 2, which has stronger assumptions and conclusions than Theorem 1. 
Fig. 1 Graphs $\Gamma_{x, y, z}($ left) and $\Gamma_{x, y, z}^{\prime}($ right $)$
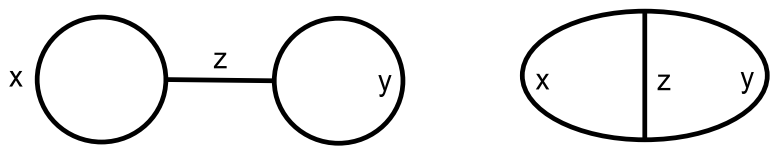

Proof For any triple of integers $x, y, z$ (with $x \geq 3, y \geq 3$ and $z \geq 0$ ) consider two graphs $\Gamma_{x, y, z}$ and $\Gamma_{x, y, z}^{\prime}$, drawn schematically on Fig. 1. The graph $\Gamma_{x, y, z}$ is topologically the union of two circles joined by an interval; the circle on the left consists of $x$ intervals, the circle on the right is subdivided into $y$ intervals, and the interval connecting them consists of $z$ subintervals. The graph $\Gamma_{x, y, z}^{\prime}$, shown schematically on the right of Fig. 1, is the union of three arcs consisting of $x, y$, and $z$ intervals. Clearly $\chi\left(\Gamma_{x, y, z}\right)=-1=\chi\left(\Gamma_{x, y, z}^{\prime}\right)$. In the case $z=0$ the corresponding interval degenerates to a point.

It is easy to see that any graph $\Gamma$ with $\chi(\Gamma)<0$ contains, as a subgraph, either $\Gamma_{x, y, z}$ or $\Gamma_{x, y, z}^{\prime}$ for some $x, y, z$.

Consider the cones $S_{x, y, z}=C\left(\Gamma_{x, y, z}\right)$ and $S_{x, y, z}^{\prime}=C\left(\Gamma_{x, y, z}^{\prime}\right)$. By the arguments leading to Corollary 13 we have

$$
\mu\left(S_{x, y, z}\right)=\mu\left(S_{x, y, z}^{\prime}\right)=1 .
$$

Applying Lemma 10 we find

$$
\mathbb{P}\left(S_{x, y, z} \rightarrow Y\right) \leq(p n)^{f}
$$

where $f=x+y+z$. Thus,

$$
\begin{aligned}
\sum_{x, y \geq 3, z \geq 0} \mathbb{P}\left(S_{x, y, z} \rightarrow Y\right) & \leq \sum_{f \geq 6} f^{2} \cdot(p n)^{f} \\
& \leq \sum_{f \geq 6}(2 p n)^{f}=\frac{(2 p n)^{6}}{1-2 p n} .
\end{aligned}
$$

We see that if $p n \rightarrow 0$, then the probability that there exist $x, y, z$ such that the 2complex $S_{x, y, z}$ admits a simplicial immersion into $Y$ tends to zero as $n \rightarrow \infty$.

Similarly, if $p n \rightarrow 0$, then the probability that there exist $x, y, z$ such that the 2-complex $S_{x, y, z}^{\prime}$ admits a simplicial immersion into $Y$ tends to zero.

Consider a vertex $v$ of the random 2-complex $Y$. The link $L_{v}$ of $v$ is a graph and the cone $C\left(L_{v}\right)$ embeds simplicially into $Y$. If for a connected component $L_{v}^{\prime}$ of $L_{v}$ one has $\chi\left(L_{v}^{\prime}\right)<0$ then for some integers $x, y, z$ the component $L_{v}^{\prime}$ contains either $\Gamma_{x, y, z}$ or $\Gamma_{x, y, z}^{\prime}$. Thus we see that $\chi\left(L_{v}^{\prime}\right)<0$ implies that for some $x, y, z$ the complex $Y$ contains either $S_{x, y, z}$ or $S_{x, y, z}^{\prime}$ as a subcomplex. Using the arguments given above, we obtain that for any vertex $v$ of $Y$, the Euler characteristic of every connected component $L_{v}^{\prime}$ of the link $L_{v}$ of $v$ satisfies

$$
\chi\left(L_{v}^{\prime}\right) \geq 0
$$

a.a.s. In other words, every connected component of the link of any vertex of $Y$ is either contractible or is homotopy equivalent to the circle.

Let $S$ be a pure and closed simplicial subcomplex of $Y$. The preceding arguments show that the link of any vertex of $S$ is a disjoint union of circles. In other words, we 
Fig. 2 2-complex $L_{x, y}$

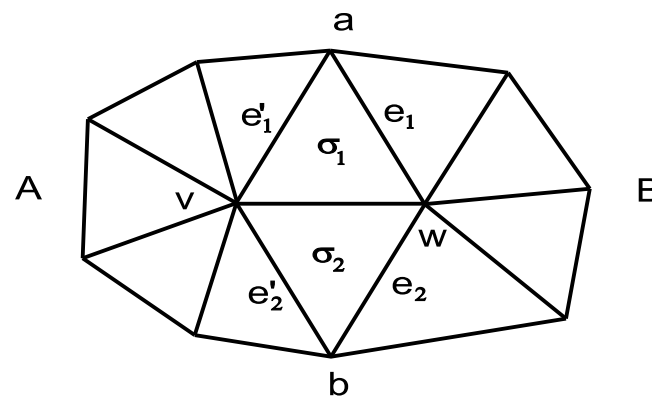

obtain that any pure closed subcomplex $S \subset Y$ is a closed pseudo-surface; i.e., every edge of $S$ is incident to exactly two 2-simplexes of $S$, a.a.s.

For any two positive integers $x, y \geq 3$ with $\max (x, y) \geq 4$, let $L_{x, y}$ be a subdivision of the disk $D^{2}$ shown on Fig. 2. The complex $L_{x, y}$ has two internal vertices $v, w$ such that the degree of $v$ is $x$ and the degree of $w$ is $y$. The total number of vertices of $L_{x, y}$ equals $x+y-2$; the number of faces of $L_{x, y}$ is also $x+y-2$; therefore $\mu\left(L_{x, y}\right)=1$.

In the special case $x=3$ and $y=3$ the complex $L_{3,3}$ is defined to be the tetrahedron with vertices $v, w, a, b$. The equality $\mu\left(L_{3,3}\right)=1$ remains true.

By Lemma 10,

$$
\mathbb{P}\left(L_{x, y} \rightarrow Y\right) \leq(p n)^{f},
$$

where $f=x+y-2$. Thus,

$$
\begin{aligned}
\sum_{x, y \geq 3} \mathbb{P}\left(L_{x, y} \rightarrow Y\right) & \leq \sum_{f \geq 4} f \cdot(p n)^{f} \\
& \leq \sum_{f \geq 4}(2 p n)^{f}=\frac{(2 p n)^{4}}{1-(2 p n)} .
\end{aligned}
$$

This shows that, if $p n \rightarrow 0$, then, with probability tending to one as $n \rightarrow \infty$, none of the complexes $L_{x, y}$ can be immersed $L_{x, y} \rightarrow Y$ into $Y$.

Next we show that for any nonempty closed pseudo-surface $S$ there exist positive integers $x, y \geq 3$ and an immersion $L_{x, y} \rightarrow S$. Consider an edge $e=v w$ of $S$ and two 2-simplexes $\sigma_{1}$ and $\sigma_{2}$ incident to it, as shown on Fig. 2. The link of $v$ in $S$ is a disjoint union of circles. It contains the edges $e_{1}$ and $e_{2}$ shown on Fig. 2. Therefore we may find a simple arc $A$ in the link of $v$ in $S$ connecting the points $a$ and $b$ and disjoint from the interior of the $\operatorname{arc} e_{1} \cup e_{2}$. Similarly, we may find a simple arc $B$ connecting $a$ and $b$ in the link of $w$ in $S$ and disjoint from the interior of $e_{1}^{\prime} \cup e_{2}^{\prime}$. Let $x$ and $y$ be such that the number of 2-simplexes in arc $A$ (correspondingly, $B$ ) is $x-2$ (correspondingly $y-2$ ). It is now obvious that we obtain an immersion of the 2-complex $L_{x, y}$ into $S$. It may not be an embedding since the images of some points of $A$ may coincide with the images of some points of $B$.

Now we see that if a random 2-complex contains a closed pseudo-surface, then there is an immersion $L_{x, y} \rightarrow Y$. 
Hence, summarizing the statements made above, we conclude that in the case $p n \rightarrow 0$, a random 2-complex contains no nonempty closed two-dimensional subcomplexes $S \subset Y$, a.a.s.

We will now use the notion of combinatorial collapse as reminded at the end of the Introduction. Consider the sequence of collapses $Y \searrow Y^{\prime} \searrow Y^{\prime \prime} \searrow \cdots$. There are two possibilities: either (a) after a finite number of collapses we obtain a closed two-dimensional complex $Y^{(k)}$; or (b) for some $k$ the complex $Y^{(k)}$ is one dimensional, i.e., a graph. Our discussion above implies that if $p n \rightarrow 0$, then for a random 2-complex $Y$ the possibility (a) happens with probability tending to 0 . Therefore, with probability tending to 1 , a random 2-complex collapses to a graph, under the assumption $p \ll n^{-1}$.

This completes the proof.

Remark 17 The main step of the preceding proof was to show that for $p \ll n^{-1}$ a random 2-complex $Y$ contains no nonempty closed two-dimensional subcomplexes $S \subset Y$. From Lemma 34 below we know that for any closed 2-complex $S$ one has $\tilde{\mu}(S) \leq 1$. Therefore, given a closed 2-complex $S$, we may apply Theorem 15 to conclude that the probability that this $S$ embeds into a random 2-complex $Y \in G\left(\Delta_{n}^{(2)}, p\right)$ tends to zero as $n \rightarrow \infty$. However, this would not be strong enough to prove Theorem 1 since we need to know (as shown in the proof above) that the probability that there exists a closed 2-complex $S$ which embeds to a random 2-complex tends to zero.

\section{Surfaces in Random 2-Complexes}

In this section we apply the results of Sect. 4 and study embeddings of triangulated surfaces into random two-dimensional complexes.

Definition 18 A finite simplicial 2-complex $S$ is called balanced if

$$
\mu(S)=\tilde{\mu}(S),
$$

i.e., if the quantities defined in Definitions 11 and 14 coincide. In other words, $S$ is balanced if

$$
\mu(S) \leq \mu\left(S^{\prime}\right)
$$

for any subcomplex $S^{\prime} \subset S$.

Definition 18 is similar to the corresponding notion for random graphs, see [13].

In this section we show that there exist many unbalanced triangulations of the disk; however, all closed triangulated surfaces are balanced. We start with the following observation.

Lemma 19 A connected simplicial 2-complex $S$ is balanced if and only if $\mu(S) \leq$ $\mu\left(S^{\prime}\right)$ for all connected subcomplexes $S^{\prime} \subset S$. 
Proof Let $S^{\prime}=S_{1}^{\prime} \sqcup S_{2}^{\prime}$ be a disjoint union of two subcomplexes. We show that

$$
\mu\left(S^{\prime}\right) \geq \min \left\{\mu\left(S_{1}^{\prime}\right), \mu\left(S_{2}^{\prime}\right)\right\}
$$

and thus $\mu(S) \leq \mu\left(S_{i}^{\prime}\right)$, where $i=1,2$, implies $\mu(S) \leq \mu\left(S^{\prime}\right)$. Let $v_{i}$ and $f_{i}$ denote the number of vertices and faces of $S_{i}^{\prime}, i=1,2$. Assume that $v_{1} / f_{1} \leq v_{2} / f_{2}$. Then one easily checks that

$$
\mu\left(S^{\prime}\right)=\frac{v_{1}+v_{2}}{f_{1}+f_{2}} \geq \frac{v_{1}}{f_{1}}=\mu\left(S_{1}^{\prime}\right) .
$$

The result now follows by induction on the number of connected components of $S^{\prime}$.

Example 20 Let $S=\Sigma_{g}$ be a triangulated closed orientable surface of genus $g \geq 0$. Then $\chi(S)=2-2 g=v-e+f$ where $v, e, f$ denote the numbers of vertices, edges, and faces in $S$ correspondingly. Each edge is contained in two faces which gives $3 f=2 e$, and therefore

$$
\mu\left(\Sigma_{g}\right)=\frac{1}{2}+\frac{2-2 g}{f} .
$$

Similarly, if $S=N_{g}$ is a triangulated closed nonorientable surface of genus $g \geq 1$ then $\chi\left(N_{g}\right)=2-g$ and

$$
\mu\left(N_{g}\right)=\frac{1}{2}+\frac{2-g}{f} .
$$

Formulae (8) and (9) give the following.

Corollary 21 The invariants $\mu\left(\Sigma_{g}\right)$ of orientable triangulated surfaces satisfy:

1. $1 / 2<\mu\left(\Sigma_{g}\right) \leq 1$ for $g=0$ (since $\left.f \geq 4\right)$;

2. $\mu\left(\Sigma_{g}\right)=1 / 2$ for $g=1$ (the torus);

3. $\mu\left(\Sigma_{g}\right)<1 / 2$ for $g>1$;

4. If $f \rightarrow \infty$ (i.e., when the surface is subsequently subdivided) then $\mu\left(\Sigma_{g}\right) \rightarrow 1 / 2$.

Corollary 22 The invariants $\mu\left(N_{g}\right)$ of nonorientable triangulated surfaces satisfy:

1. $1 / 2<\mu\left(N_{g}\right) \leq 3 / 5$ for $g=1$ (since $f \geq 10$ );

2. $\mu\left(N_{g}\right)=1 / 2$ for $g=2$ (the Klein bottle);

3. $\mu\left(N_{g}\right)<1 / 2$ for $g>2$;

4. If $f \rightarrow \infty$ (i.e., when the surface is subsequently subdivided) then $\mu\left(N_{g}\right) \rightarrow 1 / 2$.

Here we used the well-known fact that any triangulation of the real projective plane $\mathbf{R P}^{2}$ has $f \geq 10$ faces; see $[5,11,14,19]$.

Example 23 Let $S$ be a triangulated disk. Then $\chi(S)=v-e+f=1$ and $3 f=2 e-$ $e_{0}$ where $e_{0}$ is the number of edges in the boundary $\partial S$. Substituting $e=\left(3 f+e_{0}\right) / 2$, one obtains

$$
\mu(S)=\frac{1}{2}+\frac{e_{0}}{2 f}+\frac{1}{f} .
$$


Fig. 3 An $n$-gon $S$ (left) and a square with implanted $n$-gon $T$ (right)
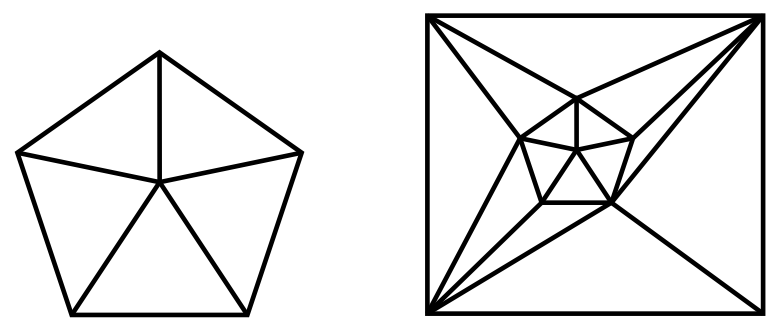

As a specific example consider the regular $n$-gon $S$ shown on Fig. 3 left. Then $v=n+1, f=n$, and

$$
\mu(S)=1+\frac{1}{n}
$$

On Fig. 3 on the right we have $e_{0}=4$ and the number of faces $f$ equals $f=$ $2 n+4$. Thus

$$
\mu(T)=\frac{1}{2}+\frac{3}{2 n+4}
$$

converges to $\frac{1}{2}$ as $n \rightarrow \infty$.

Corollary 24 For any triangulation $S$ of the disk one has $\mu(S)>1 / 2$. There exist triangulations $S$ of $D^{2}$ with $\mu(S)$ arbitrarily close to $1 / 2$.

Example 25 Let $S^{\prime}$ be such that $\mu\left(S^{\prime}\right)<1$ and suppose that $S$ is obtained from $S^{\prime}$ by adding a triangle $\Delta$ such that $S^{\prime} \cap \Delta$ is an edge. Then $S$ is not balanced. Indeed, $v_{S}=v_{S^{\prime}}+1$, and $f_{S}=f_{S^{\prime}}+1$, and

$$
\mu(S)=\frac{v_{S^{\prime}}+1}{f_{S^{\prime}}+1}>\frac{v_{S^{\prime}}}{f_{S^{\prime}}}=\mu\left(S^{\prime}\right) .
$$

Corollary 26 There exist unbalanced triangulations of the disk.

Proof Start with a disk triangulation $S^{\prime}$ with $\mu\left(S^{\prime}\right)<1$ (for instance, $S^{\prime}$ can be the square with implanted $n$-gon, see Example 23) and add a triangle $S=S^{\prime} \cup \Delta$ such that $S^{\prime} \cap \Delta$ is an edge lying in the boundary $\partial S^{\prime}$. Then $\mu(S)>\mu\left(S^{\prime}\right)$ (see Example 25) and $S$ is unbalanced. Clearly, $S$ is homeomorphic to the two-dimensional disk.

Theorem 27 Any closed connected triangulated surface $S$ is balanced.

Proof Let $S^{\prime} \subset S$ be a connected subcomplex, $S^{\prime} \neq S$. We may assume that each edge of $S^{\prime}$ belongs to either one or two triangles of $S^{\prime}$ (since any edge which is not incident to a triangle can be simply removed without affecting $\left.\mu\left(S^{\prime}\right)\right)$. Then we have

$$
\chi\left(S^{\prime}\right)=1-b_{1}\left(S^{\prime}\right)=v^{\prime}-e^{\prime}+f^{\prime},
$$


where $v^{\prime}, e^{\prime}, f^{\prime}$ are the numbers of vertices, edges, and faces in $S^{\prime}$. Here we use the assumption that $S^{\prime}$ is connected (i.e., $b_{0}\left(S^{\prime}\right)=1$ ) and $S^{\prime} \neq S$ (i.e., $b_{2}\left(S^{\prime}\right)=0$ ). One may write

$$
3 f^{\prime}=2 e^{\prime}-e_{0}
$$

where $e_{0}$ is the number of edges incident to exactly one 2-simplex. Expressing $e^{\prime}$ through $f^{\prime}$ and $e_{0}$ and substituting into (11) we obtain

$$
\mu\left(S^{\prime}\right)=\frac{1}{2}+\frac{1-b_{1}\left(S^{\prime}\right)}{f^{\prime}}+\frac{e_{0}}{2 f^{\prime}} .
$$

Assume first that $S$ is orientable and has genus $g$, i.e., $S=\Sigma_{g}$. Then we have formula (8) and the inequality $\mu\left(S^{\prime}\right) \geq \mu(S)$ is equivalent to

$$
\frac{1-b_{1}\left(S^{\prime}\right)}{f^{\prime}}+\frac{e_{0}}{2 f^{\prime}} \geq \frac{2-2 g}{f}
$$

or

$$
f\left[2-2 b_{1}\left(S^{\prime}\right)+e_{0}\right] \geq(4-4 g) f^{\prime},
$$

where $f$ denotes the number of 2 -simplexes in $S$. Since $f \geq f^{\prime}$ the above inequality follows from

$$
2-2 b_{1}\left(S^{\prime}\right)+e_{0} \geq 4-4 g .
$$

Since $b_{1}(S)=2 g$ the latter inequality is equivalent to

$$
b_{1}\left(S^{\prime}\right) \leq b_{1}(S)+e_{0} / 2-1 .
$$

The homological exact sequence of $\left(S, S^{\prime}\right)$ has the form

$$
\begin{aligned}
0 \rightarrow H_{2}(S ; \mathbf{Q}) & \rightarrow H_{2}\left(S, S^{\prime} ; \mathbf{Q}\right) \stackrel{j_{*}}{\rightarrow} H_{1}\left(S^{\prime} ; \mathbf{Q}\right) \\
& \rightarrow H_{1}(S ; \mathbf{Q}) \rightarrow H_{1}\left(S, S^{\prime} ; \mathbf{Q}\right) \rightarrow 0 .
\end{aligned}
$$

Here $H_{2}(S ; \mathbf{Q})=\mathbf{Q}$ and by the Poincaré duality theorem (see [10], Proposition 3.46)

$$
H_{2}\left(S, S^{\prime} ; \mathbf{Q}\right) \simeq H^{0}\left(S-S^{\prime} ; \mathbf{Q}\right)
$$

has dimension equal to the number $k$ of path-connected components of the complement $S-S^{\prime}$. Formally, we find a compact deformation retract $K \subset S-S^{\prime}$ such that $S-K$ deformation retracts onto $S^{\prime}$ and apply Proposition 3.46 from [10] to it; thus we obtain (14).

It follows that the image of $j_{*}$ has dimension $k-1$ and therefore the long exact sequence implies

$$
b_{1}(S) \geq b_{1}\left(S^{\prime}\right)-k+1 .
$$

Each of the connected components of the complement $S-S^{\prime}$ is bounded by a simple polygonal curve having at least 3 edges. Therefore, we see that

$$
e_{0} \geq 3 k
$$

and now (13) follows from (15). 
Consider now the case when the surface $S$ is nonorientable, $S=N_{g}$. In this case the arguments are similar, but we will consider the homology groups with coefficients in $\mathbf{Z}_{2}$ and the $\mathbf{Z}_{2}$-Betti numbers, which we will denote

$$
b_{i}^{\prime}(X)=\operatorname{dim} H_{i}\left(X ; \mathbf{Z}_{2}\right) .
$$

Comparing $\mu\left(S^{\prime}\right)$ given by (12) and $\mu(S)$ given by (9) and taking into account the equality

$$
b_{1}^{\prime}(S)=g,
$$

we see that the inequality $\mu\left(S^{\prime}\right) \geq \mu(S)$ is equivalent to

$$
b_{1}^{\prime}\left(S^{\prime}\right) \leq b_{1}^{\prime}(S)+e_{0} / 2-1,
$$

which is analogous to (13). The inequality (17) follows from arguments similar to the ones given above with $\mathbf{Z}_{2}$ coefficients replacing the rationals $\mathbf{Q}$, using the Poincaré duality and the inequality (16).

In the following statement we consider "small surfaces," i.e., triangulated surfaces which do not depend on $n$. Theorems 15 and 27 and Corollaries 21 and 22 imply the following.

\section{Corollary 28 One has:}

1. If $p \ll n^{-1}$ then a random 2-complex $Y \in G\left(\Delta_{n}^{(2)}, p\right)$ contains $^{4}$ no small ${ }^{5}$ closed surfaces, a.a.s.

2. If $n^{-1} \ll p \ll n^{-3 / 5}$ then a random 2-complex $Y$ contains small spheres but no small closed surfaces of other topological types, a.a.s.

3. If $n^{-3 / 5} \ll p \ll n^{-1 / 2}$ then a random 2-complex $Y$ contains small spheres and projective planes but no small closed surfaces of higher genera, a.a.s.

4. If $p \gg n^{-1 / 2}$ then a random 2-complex $Y$ contains all small spheres, projective planes, tori and Klein bottles, a.a.s.

5. If $p \gg n^{-1 / 2+\epsilon}$ for some $\epsilon>0$ then, given a topological type of a closed surface, there exists $f_{0}=f_{0}(\epsilon)$, such that any triangulation of the surface having more than $f_{0}$ 2-simplexes will be simplicially embeddable into a random 2-complex $Y$, a.a.s. In particular, if $p \gg n^{-1 / 2+\epsilon}$, a random 2-complex $Y$ contains small closed orientable and nonorientable surfaces of all possible topological types, a.a.s.

Proof These statements follow from Theorem 27 and formulae (8) and (9).

Statement 5 of this corollary can be compared with Theorem 3, which deals with topological embeddings.

Corollary 29 For a random 2-complex $Y \in G\left(\Delta_{n}^{(2)}, p\right)$ with $p \gg n^{-1}$ one has

$$
\pi_{2}(Y) \neq 0, \quad \text { and } \quad H_{2}(Y ; \mathbf{Z}) \neq 0
$$

a.a.s.

\footnotetext{
${ }^{4}$ In this corollary the word "contains" means "contains as a simplicial subcomplex."

5 In this statement one may remove the word "small" as follows from the proof of Theorem 1.
} 
Proof Indeed, by the previous corollary, for $p \gg n^{-1}$ a random 2-complex $Y$ contains a tetrahedron as a simplicial subcomplex. The fundamental class of this tetrahedron gives a nontrivial element of $H_{2}(Y)$. The tetrahedron can also be viewed as a sphere in $Y$ representing a nontrivial class in $\pi_{2}(Y)$.

The statement $H_{2}(Y ; \mathbf{Z}) \neq 0$ also follows from Theorem 6 and from the result of D. Kozlov [15].

\section{Remarks Concerning the Invariant $\mu(S)$}

First we observe that $\mu(S)$ admits the following curious interpretation.

For each vertex $u_{i} \in V(S)$ its degree $\operatorname{deg}\left(u_{i}\right)$ is defined as the number of edges incident to $u_{i}$. For an edge $e_{i} \in E(S)$ the degree $\operatorname{deg}\left(e_{i}\right)$ is defined as the number of two-dimensional simplexes incident to $e_{i}$. Next we define the average vertex degree and the average edge degree by the formulae

$$
D_{v}(S)=v^{-1} \cdot \sum_{u_{i} \in V(S)} \operatorname{deg}\left(u_{i}\right), \quad D_{e}(S)=e^{-1} \cdot \sum_{e_{i} \in E(S)} \operatorname{deg}\left(e_{i}\right) .
$$

Lemma 30 For any 2-complex $S$ one has

$$
\mu(S) \cdot D_{v}(S) \cdot D_{e}(S)=6 .
$$

Proof The statement follows from the definition

$$
\mu(S)=v / f=6 \cdot \frac{v}{2 e} \cdot \frac{e}{3 f}
$$

using the following obvious formulae:

$$
3 f=\sum_{e_{i} \in E(S)} \operatorname{deg}\left(e_{i}\right), \quad 2 e=\sum_{u_{i} \in V(S)} \operatorname{deg}\left(u_{i}\right) .
$$

Lemma 31 For any strongly connected 2-complex $S$ one has

$$
\mu(S) \leq 1+\frac{2}{f}
$$

where $f=f_{S}$ is the number of faces in $S$.

Proof Without loss of generality we may assume that $S$ is pure; otherwise we apply the arguments below to the pure part of $S$.

Given a pure strongly connected 2-complex $S$, there exists a sequence of subcomplexes $T_{1} \subset T_{2} \subset \cdots \subset T_{f}=S$ such that (a) each $T_{i}$ has exactly $i$ faces, i.e., $f_{T_{i}}=i$, and (b) the subcomplex $T_{i+1}$ is obtained from $T_{i}$ by adding a single 2 -simplex $\sigma_{i}$ with the property that the intersection $\sigma_{i} \cap T_{i}$ contains an edge of $\sigma_{i}$. If $v_{i}$ denotes the number of vertices of $T_{i}$ then $v_{i+1} \leq v_{i}+1$. Since $v_{1}=3$, it follows that $v=v_{f} \leq f+2$, implying (19). 
Corollary 32 Suppose that a 2-complex $S=S_{1} \cup S_{2}$ is the union of two strongly connected subcomplexes such that the intersection $S_{1} \cap S_{2}$ is at most one dimensional. (a) If $S_{1} \cap S_{2}$ contains at least 4 vertices, then $\mu(S) \leq 1$. (b) If the intersection $S_{1} \cap S_{2}$ contains $\geq 5$ vertices, then $\mu(S)<1$.

Proof Denote $v_{i}=v_{S_{i}}, f_{i}=f_{S_{i}}$, where $i=1,2$ and, as usual, $v=v_{S}, f=f_{S}$. By the previous lemma, $v_{i} \leq f_{i}+2$, and thus we obtain

$$
\begin{aligned}
\mu(S) & =\frac{v_{1}+v_{2}-v_{0}}{f_{1}+f_{2}} \leq \frac{f_{1}+2+f_{2}+2-v_{0}}{f_{1}+f_{2}} \\
& =1+\frac{4-v_{0}}{f_{1}+f_{2}},
\end{aligned}
$$

where $v_{0}$ is the number of vertices lying in the intersection $S_{1} \cap S_{2}$. Thus, $\mu(S) \leq 1$ if $v_{0} \geq 4$ and $\mu(S)<1$ if $v_{0}>4$.

Lemma 33 Let $S$ be a connected, pure, closed (i.e., $\partial S=\emptyset)$ 2-complex with $\chi(S)=$ 1 having at least 3 edges of degree $\geq 3$. Then

$$
\mu(S) \leq \frac{1}{2}-\frac{1}{2 f},
$$

where $f=f_{S}$ is the number of faces.

Proof We have

$$
v-e+f=1
$$

(since $\chi(S)=1$ ) and

$$
3 f \geq 2 e+3 .
$$

The last inequality follows from the formula

$$
3 f=2 e+e_{3}+e_{4}+\cdots,
$$

where $e_{r}$ denotes the number of edges of degree at least $r$ in $S$ with $r=3,4, \ldots$ From (23) and (24) we obtain $v \leq \frac{f}{2}-\frac{1}{2}$, implying (22).

An example of a 2-complex satisfying the condition of the previous lemma is the house with two rooms; see [10], p. 4.

Lemma 34 Let $S$ be a closed 2-complex, i.e., $\partial S=\emptyset$. Then

$$
\tilde{\mu}(S) \leq 1 .
$$

Moreover, if we additionally assume that $S$ does not contain the tetrahedron as a subcomplex, then

$$
\tilde{\mu}(S) \leq 5 / 6
$$


Proof Without loss of generality, we may assume that $S$ is connected, since otherwise we can apply the following arguments to a connected component of $S$ and use the monotonicity property (6). Moreover, we may assume that $S$ is pure, since otherwise we may deal with the maximal pure subcomplex of $S$ instead of $S$.

Suppose first that $H_{2}\left(S ; \mathbf{Z}_{2}\right)=0$. Then by the Euler-Poincaré theorem, $\chi(S) \leq 1$, and we have

$$
v-e+f=\chi(S) \leq 1, \quad \text { and } \quad 3 f \geq 2 e,
$$

where $v, e, f$ denote the numbers of vertices, edges, and faces in $S$. In the latter inequality we used the assumptions that $S$ is pure and closed. These inequalities imply that

$$
v-f / 2 \leq \chi(S) \leq 1, \quad \text { and } \quad \mu(S) \leq 1 / 2+1 / f .
$$

Since $f \geq 4$ we obtain that $\tilde{\mu}(S) \leq \mu(S) \leq 3 / 4<1$.

Assume now that $H_{2}\left(S ; \mathbf{Z}_{2}\right) \neq 0$. We will show that there is a subcomplex $S^{\prime} \subset S$ which is also closed, $\partial S^{\prime}=\emptyset$, and satisfies $H_{2}\left(S^{\prime} ; \mathbf{Z}_{2}\right)=\mathbf{Z}_{2}$. Indeed, consider a nonzero two-dimensional cycle $c=\sum_{i \in I} \sigma_{i}$ with $\mathbf{Z}_{2}$ coefficients, where the $\sigma_{i}$ are distinct 2-simplexes of $S$. Let $I^{\prime} \subseteq I$ be the minimal subset of the indexing set $I$ for which $c^{\prime}=\sum_{i \in I^{\prime}} \sigma_{i}$ is still a cycle, and let $S^{\prime}=\bigcup_{i \in I^{\prime}} \sigma_{i}$ be the corresponding subcomplex of $S$. Then clearly $H_{2}\left(S^{\prime} ; \mathbf{Z}_{2}\right)=\mathbf{Z}_{2}$ and $S^{\prime}$ is closed and pure.

By the Euler-Poincaré theorem, $\chi\left(S^{\prime}\right) \leq 2$, and we have

$$
v^{\prime}-e^{\prime}+f^{\prime}=\chi\left(S^{\prime}\right) \leq 2, \quad \text { and } 3 f^{\prime} \geq 2 e^{\prime},
$$

where $v^{\prime}, e^{\prime}, f^{\prime}$ denote the numbers of vertices, edges, and faces in $S^{\prime}$. This gives

$$
v^{\prime}-f^{\prime} / 2 \leq \chi\left(S^{\prime}\right) \leq 2,
$$

and

$$
\mu\left(S^{\prime}\right) \leq \frac{1}{2}+\frac{2}{f^{\prime}} .
$$

Since $f^{\prime} \geq 4$, the last inequality gives $\mu\left(S^{\prime}\right) \leq 1$ and we have $\tilde{\mu}(S) \leq \mu\left(S^{\prime}\right) \leq 1$. If $f^{\prime} \geq 6$ then similarly we obtain $\mu\left(S^{\prime}\right) \leq 1 / 2+2 / 6=5 / 6$ and $\tilde{\mu}(S) \leq 5 / 6$. In the remaining case $f^{\prime}=4$ the complex $S$ contains the tetrahedron as a subcomplex. Here we use the observation that there are no closed 2-complexes having 5 faces, i.e., $f^{\prime}$ cannot be equal to 5 .

\section{Topological Embeddings: Proof of Theorem 3}

Proof We show that there exists a subdivision of $S$ which simplicially embeds into $Y$ a.a.s.

We subdivide $S$ by introducing a new vertex in the center of each 2 -simplex and connecting it to three vertices, as shown on Fig. 4. We denote by $S^{\prime}$ the new triangulation. Let $v, f$ and $v^{\prime}, f^{\prime}$ denote the numbers of vertices and faces of $S$ and $S^{\prime}$ respectively. Then clearly

$$
v^{\prime}=v+f, \quad f^{\prime}=3 f .
$$


Fig. 4 A 2-simplex (left) and its subdivision (right)
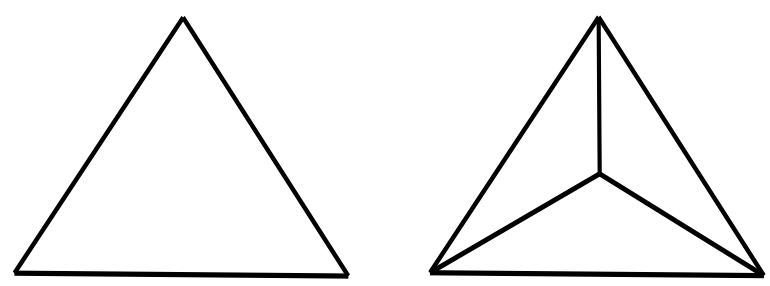

Therefore, we find that

$$
\mu\left(S^{\prime}\right)-\frac{1}{2}=\frac{1}{3}\left(\mu(S)-\frac{1}{2}\right) .
$$

We claim that a similar formula holds for $\tilde{\mu}$, i.e.,

$$
\tilde{\mu}\left(S^{\prime}\right)-\frac{1}{2}=\frac{1}{3}\left(\tilde{\mu}(S)-\frac{1}{2}\right) .
$$

Indeed, let $T \subset S$ be a subcomplex. Then its subdivision $T^{\prime}$ (defined as explained above) is a subcomplex of $S^{\prime}$, and the numbers $\mu(T)$ and $\mu\left(T^{\prime}\right)$ are related by (26). We show below that

$$
\tilde{\mu}\left(S^{\prime}\right)=\min _{T \subset S} \mu\left(T^{\prime}\right) .
$$

Clearly, (28) implies

$$
\tilde{\mu}\left(S^{\prime}\right)=\min _{T \subset S}\left[\frac{1}{3}\left(\mu(T)-\frac{1}{2}\right)+\frac{1}{2}\right]=\frac{1}{3}\left(\tilde{\mu}(S)-\frac{1}{2}\right)+\frac{1}{2}
$$

which is equivalent to (27).

To prove the formula (28) consider a subcomplex $R \subset S^{\prime}$. Each 2-simplex $\sigma$ of $S$ determines three 2-simplexes of $S^{\prime}$ which we denote by $\sigma_{1}, \sigma_{2}, \sigma_{3}$. We want to show that we may replace $R$ by a subcomplex $R_{1} \subset S^{\prime}$ such that $\mu\left(R_{1}\right) \leq \mu(R)$ and either $R_{1}$ contains all simplexes $\sigma_{1}, \sigma_{2}, \sigma_{3}$ or it contains none of them.

Suppose that $R$ contains $\sigma_{1}$ and $\sigma_{2}$ but does not contain $\sigma_{3}$. Then $R_{1}=R \cup \sigma_{3}$ has the same number of vertices and a greater number of faces, i.e., $\mu\left(R_{1}\right)<\mu(R)$.

Suppose now that $R$ contains only one simplex among the $\sigma_{i}$ 's; assume that, say, $\sigma_{1} \subset R$ and $\sigma_{2} \not \subset R$ and $\sigma_{3} \not \subset R$. (A) If $\mu(R) \geq 1 / 2$, define $R_{1}$ by $R_{1}=R \cup \sigma_{2} \cup \sigma_{3}$. Then $\mu\left(R_{1}\right) \leq \mu(R)$. (B) If $\mu(R) \leq 1$ define $R_{1}$ as $R$ with $\sigma_{1}$ removed; then $\mu\left(R_{1}\right) \leq$ $\mu(R)$. Clearly at least one of the cases (A) or (B) holds and we proceed by induction, repeating this procedure with respect to all 2-simplexes $\sigma \subset S$. Thus we see that the minimum in

$$
\tilde{\mu}\left(S^{\prime}\right)=\min _{R \subset S^{\prime}} \mu(R)
$$

is achieved on subcomplexes $R \subset S^{\prime}$ which have the form $R=T^{\prime}$ for some $T \subset S$. This completes the proof of (27).

For $r=0,1,2, \ldots$ denote by $S^{r}$ the simplicial 2-complex which is obtained from $S$ by $r$ consecutive subdivisions as above. Then from (27) we obtain 
Fig. 5 Complex $X$ (left) and its subdivision $Y_{k}$ (right)
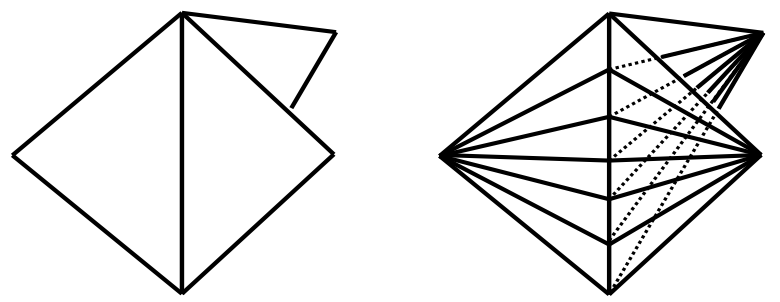

$$
\tilde{\mu}\left(S^{r}\right)-\frac{1}{2}=\frac{1}{3^{r}}\left(\tilde{\mu}(S)-\frac{1}{2}\right) .
$$

We see that this sequence approaches $1 / 2$ as $r \rightarrow \infty$. It follows that, given $\epsilon>0$, for all sufficiently large $r$ we have

$$
\tilde{\mu}\left(S^{r}\right) \geq 1 / 2-\epsilon .
$$

Thus, the assumption $p \gg n^{-1 / 2+\epsilon}$ implies $p \gg n^{-\tilde{\mu}\left(S^{r}\right)}$, and now we may apply Theorem 15 to conclude that the $r$-th subdivision $S^{r}$ simplicially embeds into $Y$, a.a.s. Hence we see that $S$ topologically embeds into $Y$, a.a.s.

Remark 35 The result of Theorem 3 cannot be improved (without adding extra hypotheses) despite a special type of subdivision used in the proof. Indeed, one sees from formulae (8) and (9) and Theorem 27 that for a closed orientable surface $\Sigma_{g}$ of genus $g \geq 1$ one has $\tilde{\mu}\left(\Sigma_{g}\right) \rightarrow 1 / 2$ as the number of 2 -simplexes $f$ goes to infinity. A similar conclusion is valid for nonorientable surfaces $N_{g}$ with $g \geq 2$.

Remark 36 Consider the following invariant $\operatorname{sign}(X) \in\{+1,-1,0\}$ of a simplicial 2-complex:

$$
\operatorname{sign}(X)=\operatorname{sign}\left(\tilde{\mu}(X)-\frac{1}{2}\right) .
$$

Formula (26) seems to suggest that it is topologically invariant. However in (26) we used a special type of subdivision. The following example shows that in general $\operatorname{sign}(X)$ is not topologically invariant. Consider the 2-complex $X$ shown on Fig. 5 (left), which is the union of three triangles having a common edge. Let $Y_{k}$ be obtained by adding $k$ new vertices along the common edge and connecting them to the remaining vertices; see Fig. 5 (right). One has $\tilde{\mu}(X)=5 / 3$ and therefore $\operatorname{sign}(X)=+1$. However,

$$
\tilde{\mu}\left(Y_{k}\right) \leq \mu\left(Y_{k}\right)=\frac{k+5}{3 k+3} .
$$

Thus, for $k>7$, one has $\tilde{\mu}\left(Y_{k}\right)<1 / 2$ and $\operatorname{sign}\left(Y_{k}\right)=-1$.

\section{Collapsibility in a Given Number of Steps}

In this section we prove Theorem 46, which gives necessary and sufficient conditions for a 2-complex to be collapsible to a graph in a prescribed number of steps. 


\subsection{Preparation}

Let $Y$ be a 2-complex, and consider the sequence of collapses

$$
Y^{(0)} \searrow Y^{(1)} \searrow Y^{(2)} \searrow \cdots \searrow Y^{(k)} \searrow \cdots .
$$

For a 2-simplex $\sigma \in Y$ define

$$
D_{Y}(\sigma)=\sup \left\{i ; \sigma \subset Y^{(i)}\right\} \in\{0,1, \ldots, \infty\} .
$$

A 2-simplex $\sigma$ is free if and only if $D_{Y}(\sigma)=0$.

A 2-complex $Y$ is collapsible to a graph in at most $k+1$ steps if and only if $D_{Y}(\sigma) \leq k$ for any 2-simplex $\sigma$. If after performing several collapses $Y^{(0)} \searrow Y^{(1)} \searrow$ $Y^{(2)} \searrow \cdots$ we obtain a subcomplex $Y^{(r)} \subset Y$ with empty boundary $\partial Y^{(r)}=\emptyset$, then $Y^{(r)}=Y^{(r+1)}=Y^{(r+2)}=\cdots$ and $D_{Y}(\sigma)=\infty$ for any simplex $\sigma$ in $Y^{(r)}$.

Lemma 37 Let $\sigma$ be a 2-simplex with $D_{Y}(\sigma)=k$ where $0<k<\infty$. Then one of the edges $e$ of $\sigma$ has the following property: for any 2-simplex $\sigma^{\prime}$ of $Y$ which is incident to $e$ and distinct from $\sigma$ one has $D_{Y}\left(\sigma^{\prime}\right)<k$ and there exists a 2-simplex $\sigma^{\prime}$ incident to $e$ and distinct from $\sigma$ such that $D_{Y}\left(\sigma^{\prime}\right)=k-1$.

Proof Since $D_{Y}(\sigma)=k$, we know that after $k$ collapses an edge $e$ of $\sigma$ becomes free. All other simplexes $\sigma^{\prime}$ of $Y$ incident to $e$ must have been eliminated in previous steps, i.e., they satisfy $D_{Y}\left(\sigma^{\prime}\right)<k$. At least one of these simplexes $\sigma^{\prime}$ must have been eliminated in step $k-1$ since otherwise $\sigma$ would have become free earlier.

Lemma 38 If $Z \subset Y$ is a subcomplex and $\sigma \subset Z$ is a 2-simplex, then

$$
D_{Z}(\sigma) \leq D_{Y}(\sigma) \text {. }
$$

Proof If a 2-simplex belongs to $Z$ and is not free in $Z$, then it is not free in $Y$. This implies that $Z^{\prime} \subset Y^{\prime}$ and therefore $Z^{(i)} \subset Y^{(i)}$ for any $i \geq 1$. Thus, the maximal $i$ such that $\sigma$ is contained in $Z^{(i)}$ is less than or equal to the maximal $i$ such that $\sigma$ is contained in $Y$, which implies the statement of the lemma.

\section{$9.2 \sigma$-Accessible Boundary}

Definition 39 Let $Y$ be a 2-complex and let $\sigma$, $\tau$ be two 2-simplexes of $Y$ with $D_{Y}(\tau)=0$ and $D_{Y}(\sigma)=k \geq 1$. A collapsing path from $\tau$ to $\sigma$ is a sequence of 2-simplexes $\tau=\sigma_{0}, \sigma_{1}, \ldots, \sigma_{k-1}, \sigma_{k}=\sigma$ such that $D_{Y}\left(\sigma_{i}\right)=i$ and each pair $\sigma_{i}$ and $\sigma_{i+1}$ has a common edge, where $i=0, \ldots, k-1$.

In a collapsing path, the initial simplex $\sigma_{0}=\tau$ is a free simplex, and hence at least one of its edges belongs to the boundary $\partial Y$.

Definition 40 Given a 2-simplex $\sigma$, we denote by $A_{Y}(\sigma) \subset \partial Y$ the union of the edges in $\sigma_{0} \cap \partial Y$ which can appear in a collapsing path $\sigma_{0}, \sigma_{1}, \ldots, \sigma_{k}$ ending at $\sigma$. We call $A_{Y}(\sigma)$ the $\sigma$-accessible part of the boundary. 
In Definition 40, clearly $k=D_{Y}(\sigma)$. Note that $A_{Y}(\sigma) \neq \emptyset$ if and only if $D_{Y}(\sigma)<\infty$.

Definition 41 Let $\sigma$ be a 2-simplex of $Y$ with $D_{Y}(\sigma) \geq 1$. For an edge $e$ of $\sigma$ define

$$
A_{Y}(\sigma, e) \subset A_{Y}(\sigma)
$$

as the set of all edges $e^{\prime}$ of the boundary $\partial Y$ with the property that there exists a collapsing path $\sigma_{0}, \sigma_{1}, \ldots, \sigma_{k}=\sigma$ such that $e^{\prime}$ is an edge of $\sigma_{0}$ and $e=\sigma_{k-1} \cap \sigma_{k}$.

If $e_{1}, e_{2}, e_{3}$ are the edges of $\sigma$, then $A_{Y}(\sigma)=\bigcup_{i=1}^{3} A_{Y}\left(\sigma, e_{i}\right)$ and the sets $A_{Y}\left(\sigma, e_{i}\right)$ need not be mutually disjoint.

Lemma 42 Let $\sigma$ and $\sigma^{\prime}$ be adjacent 2-simplexes of $Z$ with

$$
D_{Z}(\sigma)=D_{Z}\left(\sigma^{\prime}\right)+1 \text {. }
$$

Assume that any collapsing path in $Z$ ending at $\sigma$ passes through the edge $e=\sigma \cap \sigma^{\prime}$. If $Z$ is embedded as a subcomplex $Z \subset Y$ and

$$
D_{Z}\left(\sigma^{\prime}\right)<D_{Y}\left(\sigma^{\prime}\right)
$$

then

$$
D_{Z}(\sigma)<D_{Y}(\sigma)
$$

Proof Let $k=D_{Z}\left(\sigma^{\prime}\right)=D_{Z}(\sigma)-1$. We must show that $D_{Y}(\sigma) \geq k+2$. First we claim that the edge $e$ may become free only after at least $k+2$ collapses in $Y$. Assume it is free in $Y$ after $k+1$ collapses. By assumption, $D_{Y}\left(\sigma^{\prime}\right) \geq k+1$. Hence the edge $e$ can only be free after $k+1$ collapses in $Y$ if $\sigma$ has already been removed before, i.e., $D_{Y}(\sigma) \leq k$. On the other hand, by Lemma 38, $D_{Y}(\sigma) \geq D_{Z}(\sigma)=k+1$, which leads to a contradiction.

By assumption, the two edges of $\sigma$ different from $e$ are not free in $Z^{(k+1)}$ and hence they are not free in $Y^{(k+1)}$. Thus $D_{Y}(\sigma) \geq k+2$ as claimed.

Note that the assumption of Lemma 42 that any collapsing path in $Z$ ending at $\sigma$ passes through the edge $e$ is equivalent to $A_{Z}\left(\sigma, e^{\prime}\right)=\emptyset$ for the two remaining edges $e^{\prime} \neq e$ of $\sigma$.

Lemma 43 Let $Z \subset Y$ be a subcomplex. If $D_{Z}(\sigma)=D_{Y}(\sigma)$ for a 2-simplex $\sigma$ of $Z$, then there is an edge ef $\sigma$ such that

$$
\emptyset \neq A_{Z}(\sigma, e) \subset A_{Y}(\sigma, e) \subset \partial Y .
$$

Proof Without loss of generality, we may assume that $Y$ is obtained from $Z$ by attaching a single 2-simplex.

The proof is by induction on $k=D_{Y}(\sigma)=D_{Z}(\sigma)$.

In the case $k=0$, there is an edge $e$ of $\sigma$ that is free in both $Z$ and $Y$. In particular, $e \subset \partial Y$.

We include the case $k=1$. Recall that $Z^{\prime}=Z^{(1)}$ denotes the result of the first collapse of $Z, Z \searrow Z^{\prime}$. Since $D_{Z}(\sigma)=D_{Y}(\sigma)=1$, there is an edge $e$ of $\sigma$ that is 


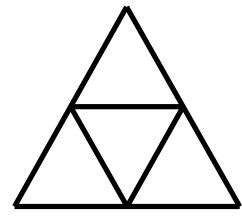

a

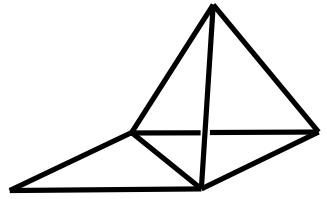

b

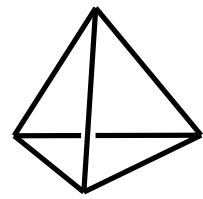

c

Fig. 6 Surfaces $\mathcal{L}_{1,2}$

free in $Y^{\prime}$ and hence in $Z^{\prime}$. Then every collapsing path $\tau, \sigma$ in $Z$ with $e=\tau \cap \sigma$ is also a collapsing path in $Y$. Hence $A_{Z}(\sigma, e) \subset A_{Y}(\sigma, e)$.

For the general case, assume that $D_{Y}(\sigma)=D_{Z}(\sigma)=k$. After $k$ collapses

$$
Z \searrow Z^{(1)} \searrow \cdots \searrow Z^{(k)}, \quad Y \searrow Y^{(1)} \searrow \cdots \searrow Y^{(k)},
$$

the 2-simplex $\sigma$ is exposed in both $Z^{(k)}$ and $Y^{(k)}$. Thus, $\sigma$ has a free edge $e$ in $Y^{(k)}$ (and hence in $Z^{(k)}$ as well). Writing $Z^{\prime}=Z^{(1)}$ and $Y^{\prime}=Y^{(1)}$, by induction, we have $\emptyset \neq A_{Z^{\prime}}(\sigma, e) \subset A_{Y^{\prime}}(\sigma, e)$ so that any collapsing path $\sigma_{1}, \ldots, \sigma_{k}$ from $\sigma_{1}=\sigma^{\prime} \subset$ $A_{Z^{\prime}}(\sigma, e)$ to $\sigma_{k}=\sigma$ in $Z^{\prime}$ is also a collapsing path in $Y^{\prime}$. Note in particular that every edge of $\sigma^{\prime}$ that is free in $Z^{\prime}$ is also free in $Y^{\prime}$. Consequently, for every free triangle $\tau$ in $Z$ which meets $\sigma^{\prime}$ in an edge free in $Z^{\prime}$, the collapsing path $\tau=\sigma_{0}, \sigma_{1}, \ldots, \sigma_{k}$ in $Z$ is a collapsing path in $Y$. The result follows.

Corollary 44 Let $Z \subset Y$ be 2-complexes such that for a 2-simplex $\sigma$ of $Z$ none of the edges $e \in A_{Z}(\sigma) \subset \partial Z$ is free in $Y$. Then

$$
D_{Z}(\sigma)+1 \leq D_{Y}(\sigma) .
$$

Proof For a contradiction, assume that $D_{Y}(\sigma) \leq D_{Z}(\sigma)$. Then $D_{Y}(\sigma)=D_{Z}(\sigma)$ by Lemma 38. We may now apply Lemma 43, which claims that there is an edge $e$ of $\sigma$ for which $\emptyset \neq A_{Z}(\sigma, e) \subset A_{Y}(\sigma, e) \subset \partial Y$. This contradicts our assumption that no edge in $A_{Z}(\sigma)$ lies on the boundary $\partial Y$.

\subsection{The List of Forbidden $r$-Pseudo-surfaces $\mathcal{L}_{k, r}$}

For a pair of integers $k=0,1, \ldots$, and $r=2,3, \ldots$ we denote by $\mathcal{L}_{k, r}$ the set of all isomorphism types of $r$-pseudo-surfaces $S$ with the following properties:

(a) Each $S \in \mathcal{L}_{k, r}$ has a specified 2-simplex $\sigma_{*}$ (called the center).

(b) If $\partial S \neq \emptyset$ then $D_{S}\left(\sigma_{*}\right)=k$.

(c) $d_{S}\left(\sigma_{*}, \sigma\right) \leq k$ for any 2-simplex $\sigma$.

Note that $\mathcal{L}_{0, r}=\{S\}$ consists of a single complex $S=\sigma_{*}$ (the triangle).

The set $\mathcal{L}_{1,2}$ consists of the three surfaces shown on Fig. 6. Each of the surfaces $\mathrm{a}, \mathrm{b}, \mathrm{c}$ is a union of 4 triangles. The surface $\mathrm{c}$ is a tetrahedron, $\mathrm{b}$ is a tetrahedron with one face open, and surface a is a fully flattened tetrahedron.

It is clear that $\mathcal{L}_{k, r}$ is finite and $\mathcal{L}_{k, r} \subset \mathcal{L}_{k, r+1}$. 
Fig. 7 Surfaces $S_{k} \in \mathcal{L}_{k, 2}$

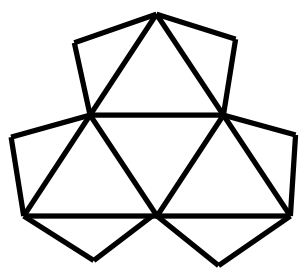

$\mathrm{S}_{2}$

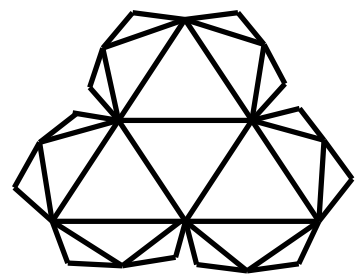

$\mathrm{S}_{3}$

Example 45 Consider the following important family of surfaces $S_{k} \in \mathcal{L}_{k, 2}$ where $k=0,1,2, \ldots$. The first surface $S_{0}$ is defined as a single triangle $S_{0}=\sigma_{*}$. The next surface $S_{1}$ is the one shown on Fig. 6a. Surfaces $S_{2}$ and $S_{3}$ are shown on Fig. 7. In general, the surface $S_{k}$ is obtained from $S_{k-1}$ by adding a triangle to every edge of the boundary $\partial S_{k-1}$. It is clear that for the central triangle $\sigma_{*}$ of $S_{k}$, one has $D_{S_{k}}\left(\sigma_{*}\right)=k$. Thus $S_{k}$ is not collapsible to a graph in $k$ steps, but is collapsible in $k+1$ steps.

It is interesting to note that the surfaces $S_{k}$ are "extra-special" triangulations of the disk in the sense of Papadima-Suciu [18], where they are studied for completely different reasons.

The following pure topological theorem plays a key role in the rest of this paper.

Theorem 46 A 2-complex $Y$ of degree at most $r \geq 2$ is not collapsible to a graph in $k$ steps, where $k=0,1,2, \ldots$, if and only if there is a surface $S \in \mathcal{L}_{k, r}$ which admits a simplicial embedding $S \rightarrow Y$.

In the proof, we will use the following statement.

Lemma 47 Let $Y$ be a finite two-dimensional simplicial complex of degree at most $r$ and let $\sigma$ be a 2-simplex in $Y$ with $D_{Y}(\sigma)=k$, where $k=0,1,2, \ldots$ Then there exists a surface $S \in \mathcal{L}_{k, r}$ and a simplicial embedding $S \rightarrow Y$ such that the central simplex $\sigma_{*}$ of $S$ is mapped onto $\sigma$.

Proof of Lemma 47 We will use induction on $k=D_{Y}(\sigma)$. For $k=0$, the statement is obvious. Assume that it is true for all cases with $D_{Y}(\sigma)<k$, and consider the situation when $D_{Y}(\sigma)=k>0$. If $Y \searrow Y^{\prime}$ is the first collapse, then $\sigma \subset Y^{\prime}$ and clearly

$$
D_{Y^{\prime}}(\sigma)=k-1
$$

and $Y^{\prime}$ has degree at most $r$. By the inductive hypothesis, there exists $S^{\prime} \in \mathcal{L}_{k-1, r}$ and a simplicial embedding $S^{\prime} \rightarrow Y^{\prime}$, mapping the central simplex of $S^{\prime}$ onto $\sigma$.

For each edge $e$ lying in $A_{S^{\prime}}(\sigma)$ choose a 2-simplex $\sigma_{e} \subset Y$ as follows. If $e \subset \partial Y^{\prime}$, let $\sigma_{e}$ be any free triangle in $Y$ containing $e$. If $e \not \subset \partial Y^{\prime}$, let $\sigma_{e}$ be any triangle in $Y^{\prime}$ containing $e$ which is not in $S^{\prime}$; such $\sigma_{e}$ exists since $e \not \subset \partial Y^{\prime}$.

Next we define a subcomplex $S \subset Y$ as the union

$$
S=S^{\prime} \cup \bigcup_{e} \sigma_{e} \subset Y,
$$


where $e$ runs over the edges in $A_{S^{\prime}}(\sigma)$. Note that $S$ is finite, pure, and strongly connected since $S^{\prime}$ is an $r$-pseudo-surface. Moreover, the degree of $S$ is at most $r$ since it is a subcomplex of $Y$. One has $D_{S}(\sigma) \geq k$ by Corollary 44. More precisely, we obtain that $D_{S}(\sigma)=k$ by Lemma 38. Finally, we observe that obviously $d_{S}\left(\sigma, \sigma^{\prime}\right) \leq k$ for any 2 -simplex $\sigma^{\prime}$ of $S$. Thus, $S \in \mathcal{L}_{k, r}$.

Proof of Theorem 46 Consider the sequence of successive collapses

$$
Y \searrow Y^{(1)} \searrow Y^{(2)} \searrow Y^{(3)} \searrow \cdots \text {. }
$$

We assume that $Y$ is not collapsible to a graph in $k$ steps, which implies that there are two possibilities: either (a) $Y^{(i)} \neq Y^{(i+1)}$ for any $i<k$; or (b) for some $i<k$, one has $\partial Y^{(i)}=\emptyset$.

In case (a), the complex $Y$ contains a 2-simplex with $D_{Y}(\sigma)=k$ and Lemma 47 gives us an embedding of an $r$-pseudo-surface $S \in \mathcal{L}_{k, r}$ into $Y$.

In case (b), we have $\partial Y^{(i)}=\emptyset$ for some $i<k$. Fix a 2-simplex $\sigma_{*} \in Y^{(i)}$ and consider distances $d_{Y^{(i)}}\left(\sigma_{*}, \sigma\right)$ to various 2-simplexes $\sigma$ of $Y^{(i)}$. If all these distances are less than or equal to $k$, then $Y^{(i)}$ belongs to $\mathcal{L}_{k, r}$ and we are done. If there are simplexes $\sigma$ such that $d_{Y^{(i)}}\left(\sigma_{*}, \sigma\right)>k$, then consider the subcomplex $Z \subset Y^{(i)}$ defined as the union of all $\sigma$ with $d_{Y^{(i)}}\left(\sigma_{*}, \sigma\right) \leq k$.

Clearly $Z$ is not collapsible to a graph in $k$ steps. Therefore, in the sequence of collapses $Z \searrow Z^{(1)} \searrow Z^{(2)} \searrow Z^{(3)} \searrow \cdots$, we again have either case (a) or (b) as above. In case (a), we apply Lemma 47; and in case (b), we obtain a subcomplex $S \subset Z$ with $\partial S=\emptyset$ such that $d\left(\sigma_{*}, \sigma\right) \leq k$ for any $\sigma \subset S$. We have $S \in \mathcal{L}_{k, r}$ in either case, completing the proof.

\section{Collapsibility of a Random 2-Complex to a Graph in a Prescribed Number of Steps}

\subsection{The Degree Sequence}

Recall that the degree of an edge $e$ in a 2-complex is defined as the number of 2-simplexes which contain $e$. The degree of an edge in a random 2-complex $Y \in G\left(\Delta_{n}^{(2)}, p\right)$ is an integer in the set $\{0,1, \ldots, n-2\}$.

Let $X_{k}: G\left(\Delta_{n}^{(2)}, p\right) \rightarrow \mathbf{Z}$ be the random variable counting the number of edges of degree $k$ in a random 2-complex, where $k=0,1,2, \ldots, n-2$. A straightforward calculation reveals that

$$
\mathbb{E}\left(X_{k}\right)=\left(\begin{array}{l}
n \\
2
\end{array}\right)\left(\begin{array}{c}
n-2 \\
k
\end{array}\right) p^{k}(1-p)^{n-2-k} .
$$

The expectation of the number of edges of degree at least $r$ in a random 2-complex is

$$
\sum_{k=r}^{n-2} \mathbb{E}\left(X_{k}\right) \leq n^{2} \sum_{k=r}^{n-2}(p n)^{k} \leq \frac{n^{2}(p n)^{r}}{1-p n} .
$$


Corollary 48 The probability that a random 2-complex $Y \in G\left(\Delta_{n}^{(2)}, p\right)$ has an edge of degree at least $r$ is less than or equal to

$$
\frac{n^{2+r} p^{r}}{1-p n} \text {. }
$$

Thus, if

$$
p \ll n^{-1-\frac{2}{r}},
$$

then a random 2-complex $Y \in G\left(\Delta_{n}^{(2)}\right.$, p) has no edges of degree $r$ or greater, a.a.s.

Proof This follows from inequality (30) by applying the first moment method; see, for instance, [13].

Next, we examine the $\tilde{\mu}$ invariants of 2-complexes $S \in \mathcal{L}_{k, r}$.

10.2 The Threshold for $k$-Collapsibility

Definition 49 Let $\tilde{\mu}_{k, r}$ denote the largest possible value of the invariant $\tilde{\mu}(S)$ for $S$ a forbidden $r$-pseudo-surface,

$$
\tilde{\mu}_{k, r}=\max _{S \in \mathcal{L}_{k, r}} \tilde{\mu}(S) \in \mathbf{Q} .
$$

For instance, examining the surfaces shown on Fig. 6 reveals that $\tilde{\mu}_{1,2}=3 / 2$.

Theorem 50 Consider a random 2-complex $Y \in G\left(\Delta_{n}^{(2)}, p\right)$.

(a) Iffor some $r \geq 2$ and $k \geq 1$, one has

$$
p \ll n^{-1-\frac{2}{r+1}} \text { and } p \ll n^{-\tilde{\mu}_{k, r}},
$$

then $Y$ is collapsible to a graph in at most $k$ steps, a.a.s.

(b) If for some $r \geq 2$ and $k \geq 1$, one has $p \gg n^{-\tilde{\mu}_{k, r}}$, then $Y$ is not collapsible to a graph in $k$ or fewer steps, a.a.s.

Proof By Corollary 48, if $p \ll n^{-1-\frac{2}{r+1}}$, then a random 2-complex $Y \in G\left(\Delta_{n}^{(2)}, p\right)$ has degree at most $r$, a.a.s. Next, we apply Theorem 46 and examine the embeddability of complexes $S \in \mathcal{L}_{k, r}$ into $Y$. By Theorem 15(A), if $p \ll n^{-\tilde{\mu}(S)}$, then $S$ does not embed into $Y$, a.a.s. Since $\tilde{\mu}_{k, r} \geq \tilde{\mu}(S)$, we see that the assumption $p \ll n^{-\tilde{\mu}_{k, r}}$ implies that no $S \in \mathcal{L}_{k, r}$ can be embedded into $Y$, a.a.s. Thus, by Theorem 46 , we see that $Y$ is collapsible to a graph in $k$ or fewer steps. This proves part (a).

To prove part (b), we apply Theorem 15 (B) to conclude that if $p \gg n^{-\tilde{\mu}_{k, r}}$, then there exists $S \in \mathcal{L}_{k, r}$ which is embeddable into $Y$, a.a.s. This implies that $Y$ is not collapsible to a graph in at most $k$ steps, a.a.s.

Lemma 51 If $S \in \mathcal{L}_{k, r}$ for some $k \geq 0, r \geq 2$ then one has

$$
\tilde{\mu}(S) \leq 1+\frac{2}{k+1} .
$$


Proof If $S$ is closed the result follows from Lemma 34. Assume now that $\partial S \neq \emptyset$. Let $\sigma_{*}$ be the central simplex of $S$ and let $\sigma_{0}, \sigma_{1}, \ldots, \sigma_{k}=\sigma_{*}$ be a collapsing path leading to $\sigma_{*}$. Here $D_{S}\left(\sigma_{i}\right)=i$ and $\sigma_{i} \cap \sigma_{i+1}$ is an edge; see Definition 40. Then the union $S^{\prime}=\bigcup_{i=0}^{k} \sigma_{i}$ is a subcomplex having exactly $k+1$ faces and at most $k+3$ vertices. Thus,

$$
\mu\left(S^{\prime}\right) \leq \frac{k+3}{k+1}=1+\frac{2}{k+1}
$$

establishing (31).

Example 52 Consider the surface $S_{k} \in \mathcal{L}_{k, 2}$ introduced in Example 45. Note that $S_{k} \in \mathcal{L}_{k, r}$ for any $r \geq 2$. The numbers of vertices $v_{k}$ and faces $f_{k}$ of $S_{k}$ satisfy the recurrence relations

$$
v_{k}=2 \cdot v_{k-1} \text { and } f_{k}=v_{k-1}+f_{k-1} .
$$

Indeed, viewing $S_{k-1}$ as a subcomplex of $S_{k}$, we see that all vertices of $S_{k-1}$ lie on the boundary, and each edge of the boundary of $S_{k-1}$ adds a vertex to $S_{k}$. This explains the first equation. For the second, note that the number of new triangles in $S_{k}$ is equal to the number of edges on $\partial S_{k-1}$.

Since $v_{0}=3$ and $f_{0}=1$, solving the recurrence relations (32) yields

$$
v_{k}=3 \cdot 2^{k} \text { and } f_{k}=3 \cdot 2^{k}-2 \text {. }
$$

Consequently,

$$
\mu\left(S_{k}\right)=1+\frac{1}{3 \cdot 2^{k-1}-1} .
$$

Lemma 53 The surface $S_{k}$ is balanced, and hence

$$
\tilde{\mu}\left(S_{k}\right)=\mu\left(S_{k}\right)=1+\frac{1}{3 \cdot 2^{k-1}-1} .
$$

Proof Let $S$ be a pure subcomplex of $S_{k}$ with $v=v(S)$ vertices and $f=f(S)$ faces. Write $v=v_{k}-m$ and $f=f_{k}-n$, where $v_{k}$ and $f_{k}$ are as above and $m$ and $n$ are the number of vertices and faces which are in $S_{k}$, but not in $S$. We claim that $m=v_{k}-v \leq f_{k}-f=n$. This assertion is established by induction.

The case $k=0$ is trivial. So assume inductively that for any $i<k$ and $S^{\prime} \subset S_{i}$ a pure subcomplex, we have $v\left(S_{i}\right)-v\left(S^{\prime}\right) \leq f\left(S_{i}\right)-f\left(S^{\prime}\right)$.

For a pure subcomplex $S \subset S_{k}$ as above, let $S^{\prime}$ be the pure part of $S \cap S_{k-1}$. Then, $m=m^{\prime}+m^{\prime \prime}$ and $n=n^{\prime}+n^{\prime \prime}$, where $v\left(S^{\prime}\right)=v_{k-1}-m^{\prime}, f\left(S^{\prime}\right)=f_{k-1}-n^{\prime}, m^{\prime \prime}$ is the number of vertices in $S_{k} \backslash S_{k-1}$ which are not in $S$, and $n^{\prime \prime}$ is the number of faces in $S_{k} \backslash S_{k-1}$ which are not in $S$.

We have $m^{\prime} \leq n^{\prime}$ by induction. Observe that the vertices of $S_{k} \backslash S_{k-1}$ are in oneto-one correspondence with the faces of $S_{k} \backslash S_{k-1}$. If such a vertex is not in $S$, then the corresponding face cannot be in $S$ either. Consequently, $m^{\prime \prime}=n^{\prime \prime}$, and $m=$ $m^{\prime}+m^{\prime \prime} \leq n^{\prime}+n^{\prime \prime}=n$, completing the proof of the claim.

It follows immediately that $\mu(S) \geq \mu\left(S_{k}\right)=\mu_{k}$. Indeed,

$$
\frac{v}{f}-\frac{v_{k}}{f_{k}}=\frac{v_{k}-m}{f_{k}-n}-\frac{v_{k}}{f_{k}}=\frac{n v_{k}-m f_{k}}{f_{k}\left(f_{k}-n\right)}=\frac{\mu_{k} n-m}{f_{k}-n} \geq \frac{n-m}{f_{k}-n} \geq 0 .
$$


Thus, $S_{k}$ is balanced.

From Lemmas 51 and 53 we obtain the following.

Corollary 54 For any $r \geq 2$ and $k \geq 0$, one has the following inequalities:

$$
1+\frac{1}{3 \cdot 2^{k-1}-1} \leq \tilde{\mu}_{k, r} \leq 1+\frac{2}{k+1} .
$$

Note that the obtained upper and lower bounds for $\tilde{\mu}_{k, r}$ are independent of $r$. We believe that $\tilde{\mu}_{k, r}=1+1 /\left(3 \cdot 2^{k-1}-1\right)$.

Proof of Theorem 2 Theorem 2 stated in the Introduction is now an immediate consequence of Theorem 50 and Corollary 54, as we explain below.

(a) Assume that $p \ll n^{-1-2 /(k+1)}$ for some $k \geq 1$. According to Corollary $54, \tilde{\mu}_{k, r} \leq$ $1+2 /(k+1)$. Choosing $r=\max (2, k)$, it then follows from Theorem 50(a) that $Y \in G\left(\Delta_{n}^{(2)}, p\right)$ is collapsible to a graph in at most $k$ steps, a.a.s.

(b) Assume that $p \gg n^{-1-1 /\left(3 \cdot 2^{k-1}-1\right)}$ for some $k \geq 1$. Then by Theorem 15 and Lemma 53 the surface $S_{k}$ (see Example 45) embeds into $Y$, a.a.s. Since $S_{k}$ cannot be collapsed to a graph in $k$ or fewer steps, we obtain that $Y$ is not collapsible to a graph in $k$ or fewer steps.

Acknowledgements D. Cohen was partially supported by Louisiana Board of Regents grant NSF(2010)PFUND-171 and National Security Agency grant H98230-11-1-0142. M. Farber was partly supported by a grant from the EPSRC. T. Kappeler was partly supported by the Swiss National Science Foundation.

This research was implemented during visits of M. Farber to FIM ETH Zürich and Louisiana State University, and visits of D. Cohen and A. Costa to the University of Zürich. Portions of this work were carried out during the Spring of 2010, when the first and the third authors participated in the Mathematisches Forschungsinstitut Oberwolfach Research in Pairs program. We thank the FIM ETH, LSU, the University of Zürich, and the MFO for their support and hospitality, and for providing productive mathematical environments.

\section{References}

1. Aronshtam, L., Linial, N., Łuczak, T., Meshulam, R.: Vanishing of the top homology of a random complex. arXiv:1010.1400

2. Babson, E., Hoffman, C., Kahle, M.: The fundamental group of random 2-complexes. J. Am. Math. Soc. 24, 1-28 (2011)

3. Cohen, D., Farber, M., Kappeler, T.: The homotopical dimension of random 2-complexes. arXiv: $1005.3383 v 1(2010)$

4. Costa, A., Farber, M., Kappeler, T.: Topology of random 2-complexes. arXiv:1006.4229v2 (2010)

5. Hartsfield, N., Ringel, G.: Clean triangulations. Combinatorica 11, 145-155 (1991)

6. Erdős, P., Rényi, A.: On the evolution of random graphs. Publ. Math. Inst. Hung. Acad. Sci. 5, 17-61 (1960)

7. Farber, M.: Topology of random linkages. Algebr. Geom. Topol. 8, 155-171 (2008)

8. Farber, M., Kappeler, T.: Betti numbers of random manifolds. Homol. Homotopy Appl. 10(1), 205222 (2008)

9. Farber, M., Schuetz, D.: Novikov-Betti numbers and the fundamental group. Russ. Math. Surv. 61, 1173-1175 (2006) 
10. Hatcher, A.: Algebraic Topology. Cambridge University Press, Cambridge (2002)

11. Heawood, P.J.: Map-colour theorem. Q. J. Pure Appl. Math. 24, 332-338 (1890)

12. Hog-Angeloni, C., Metzler, W., Sieradski, A.: Two-Dimensional Homotopy and Combinatorial Group Theory. London Math. Soc. Lecture Note Ser., vol. 197. Cambridge University Press, Cambridge (1993)

13. Janson, S., Łuczak, T., Ruciński, A.: Random Graphs. Wiley-Intersci. Ser. Discrete Math. Optim. Wiley-Interscience, New York (2000)

14. Jungerman, M., Ringel, G.: Minimum triangulations on orientable surfaces. Acta Math. 145, 121-154 (1980)

15. Kozlov, D.: The threshold function for vanishing of the top homology group of random $d$-complexes. Proc. Am. Math. Soc. 138, 4517-4527 (2010)

16. Linial, N., Meshulam, R.: Homological connectivity of random 2-complexes. Combinatorica 26, 475487 (2006)

17. Meshulam, R., Wallach, N.: Homological connectivity of random $k$-complexes. Random Struct. Algorithms 34, 408-417 (2009)

18. Papadima, S., Suciu, A.: Algebraic invariants for Bestvina-Brady groups. J. Lond. Math. Soc. 76, 273-292 (2007)

19. Ringel, G.: Wie man die geschlossenen nichtorientierbaren Flächen in möglichst wenig Dreiecke zerlegen kann. Math. Ann. 130, 317-326 (1955)

20. Shiryaev, A.N.: Probability, 2nd edn. Graduate Texts in Mathematics, vol. 95. Springer, New York (1996) 